\title{
Endogenous Activation of Kainate Receptors Regulates Glutamate Release and Network Activity in the Developing Hippocampus
}

\author{
Sari E. Lauri, ${ }^{1}$ Mikael Segerstråle, ${ }^{1}$ Aino Vesikansa, ${ }^{1}$ Francois Maingret, ${ }^{2}$ Christophe Mulle, ${ }^{3}$ Graham L. Collingridge, ${ }^{2}$ \\ John T. R. Isaac, ${ }^{2,4}$ and Tomi Taira ${ }^{1}$ \\ ${ }^{1}$ Neuroscience Center and Department of Biological and Environmental Sciences, University of Helsinki, 00014 Helsinki, Finland, ${ }^{2}$ Medical Research \\ Council Centre for Synaptic Plasticity, Department of Anatomy, Medical School, University of Bristol, Bristol BS8 1TD, United Kingdom, ${ }^{3}$ Laboratoire \\ Physiologie Cellulaire de la Synapse, Centre National de la Recherche Scientifique, Unité Mixte de Recherche 5091, Institut Francois Magendie, Université \\ Bordeaux 2, 33076 Bordeaux Cedex, France, and ${ }^{4}$ National Institutes of Health-National Institute of Neurological Disorders and Stroke, Bethesda, \\ Maryland 20892
}

\begin{abstract}
Kainate receptors (KARs) are highly expressed throughout the neonatal brain, but their function during development is unclear. Here, we show that the maturation of the hippocampus is associated with a switch in the functional role of presynaptic KARs. In a developmental period restricted to the first postnatal week, endogenous L-glutamate tonically activates KARs at CA3 glutamatergic synapses to regulate release in an action potential-independent manner. At synapses onto pyramidal cells, KARs inhibit glutamate release via a G-protein and PKC-dependent mechanism. In contrast, at glutamatergic terminals onto CA3 interneurons, presynaptic KARs can facilitate release in a G-protein-independent mechanism. In both cell types, however, KAR activation strongly upregulates inhibitory transmission. We show that, through the interplay of these novel diverse mechanisms, KARs strongly regulate the characteristic synchronous network activity observed in the neonatal hippocampus. By virtue of this, KARs are likely to play a central role in the development of hippocampal synaptic circuits.
\end{abstract}

Key words: development; interneuron; presynaptic; plasticity; pyramidal cell; synaptic transmission

\section{Introduction}

Presynaptic kainate receptors (KARs) regulating neurotransmitter release have been identified at a number of synapses in the CNS. Numerous studies have shown that pharmacological activation of these receptors can either facilitate or depress release (for review, see Kullmann, 2001; Kamiya, 2002; Lerma, 2003). Far fewer studies have investigated the physiological activation of KARs by endogenous L-glutamate. Best studied is the mossy fiber-CA3 synapse in the hippocampus; here KARs contribute to the frequency facilitation of EPSCs (Lauri et al., 2001a,b, 2003a; Schmitz et al., 2001) and are critical for the induction of longterm potentiation (Bortolotto et al., 1999; Contractor et al., 2001; Lauri et al., 2001a, 2003a; Schmitz et al., 2003). Heterosynaptic activation of KARs may also depress mossy fiber transmission (Schmitz et al., 2000) (but see Bortolotto et al., 2003). Synaptically released glutamate can also activate presynaptic KARs to inhibit glutamatergic synapses in the barrel cortex (Kidd et al.,

\footnotetext{
Received Sept. 30, 2004; revised Feb. 23, 2005; accepted March 18, 2005.

This work was supported by the Academy of Finland (T.T. and S.E.L.), The Sigrid Juselius Foundation (T.T.), The Biocentrum Helsinki (S.E.L.), the Medical Research Council (G.L.C.), the National Institute of Neurological Disorders and Stroke (J.T.R.I.), and The Wellcome Trust (F.M. and J.T.R.I.). We thank Eli Lilly and Company for providing LY382884.

Correspondence should be addressed to T. Taira at the above address. E-mail: tomi.taira@helsinki.fi. DOI:10.1523/JNEUROSCI.4050-04.2005

Copyright $\odot 2005$ Society for Neuroscience $\quad$ 0270-6474/05/254473-12\$15.00/0
}

2002) and bidirectionally regulate excitatory transmission in the cerebellum (Delaney and Jahr, 2002). It may also act heterosynaptically on KARs regulating GABAergic transmission in the hippocampus (Cossart et al., 2001; Jiang et al., 2001; Semyanov and Kullmann, 2001). By regulating dynamic properties of both glutamatergic and GABAergic synapses, presynaptic KARs are therefore likely to be critical in controlling information transfer in neuronal circuitry. However, it is unclear what the physiological consequences of KAR activation are for the activity and excitability of neuronal networks.

Spontaneous activity is a characteristic feature of immature neuronal networks and is thought to play an important role in controlling the development of synaptic circuitry (for review, see Zhang and Poo, 2001). In the neonatal hippocampus, spontaneous network bursts are seen both in vitro (Ben-Ari et al., 1989; Garaschuk et al., 1998; Palva et al., 2000) and in vivo (Lahtinen et al., 2002; Leinekugel et al., 2002). This activity consists of glutamate receptor (GluR)-driven synchronous bursts, which are rhythmically paced by $\mathrm{GABA}_{\mathrm{A}}$ receptor-mediated conductances (Khazipov et al., 1997; Bolea et al., 1999; Lamsa et al., 2000).

The GluR5 KAR subunit is highly expressed in the hippocampus during early postnatal development (Bahn et al., 1994; Ritter et al., 2002); however, data about its function are scarce. We now report that these KARs can strongly regulate both GABAergic and glutamatergic synaptic transmission in the neonatal hippocam- 
pus and control spontaneous network activity. We also show that, in the neonatal hippocampus, unlike in adults, presynaptic KARs regulating glutamatergic transmission are continually activated by ambient L-glutamate. KARs tonically inhibit or facilitate glutamate release at synapses onto pyramidal cells and stratum lucidum interneurons, respectively. These findings demonstrate novel roles for KARs in synaptic function and in network activity and suggest a critical role for KARs in the development of the hippocampal network.

\section{Materials and Methods}

Acute hippocampal slices were cut from the brain of neonatal [postnatal day 3 (P3) to P6] or young (P14-P16) rats or wild-type and GluR5 ${ }^{-1-}$ mice using standard methods. Briefly, the brain was quickly dissected into ice-cold solution containing the following (in mM): $124 \mathrm{NaCl}, 3 \mathrm{KCl}$, $1.25 \mathrm{NaH}_{2} \mathrm{PO}_{4}, 10 \mathrm{MgSO}_{4}, 26 \mathrm{NaHCO}_{3}, 10-15$ D-glucose, and $1 \mathrm{CaCl}_{2}$ (bubbled with $5 \% \mathrm{CO}_{2} / 95 \% \mathrm{O}_{2}$ ). A tissue block containing the hippocampi was dissected and glued into the stage of a vibratome (Vibratome, St. Louis, MO). Slices (400-600 $\mu \mathrm{m}$ thick) were cut transversally or sagittally in the above solution and stored at room temperature in a solution containing the following (in $\mathrm{mM}$ ): $124 \mathrm{NaCl}, 3 \mathrm{KCl}, 1.25$ $\mathrm{NaH}_{2} \mathrm{PO}_{4}, 4 \mathrm{MgSO}_{4}, 26 \mathrm{NaHCO}_{3}, 10-15$ D-glucose, and $2 \mathrm{CaCl}_{2}(5 \%$ $\mathrm{CO}_{2} / 95 \% \mathrm{O}_{2}$ ).

The slices were used $1-4 \mathrm{~h}$ after cutting, except for the experiments in which pertussis toxin (PTX) was used. For these experiments, after 30 min of recovery, the slices ( $400 \mu \mathrm{m}$; P3-P6) were washed with $1 \mathrm{ml}$ of a modified slice culture medium, consisting of $75 \%$ minimal essential medium supplemented with HEPES and bicarbonate (Invitrogen, Carlsbad, CA), 25\% Eagle's balanced salt solution (Invitrogen), 2 mm L-glutamine, and $6.5 \mathrm{mg} / \mathrm{ml}$ glucose. The slices were then placed into Millicell CM membranes (Millipore, Bedford, MA) in six-well culture trays with $1 \mathrm{ml}$ of the above medium with or without pertussis toxin $(5 \mu \mathrm{g} / \mathrm{ml})$ and transferred into a $\mathrm{CO}_{2}$ incubator $\left(35^{\circ} \mathrm{C}\right.$ under $5 \% \mathrm{CO}_{2}$ in air) for $12-15 \mathrm{~h}$.

For electrophysiological recordings, the slices were placed in a submerged recording chamber and perfused with extracellular solution containing the following (in mM): $124 \mathrm{NaCl}, 3 \mathrm{KCl}, 1.25 \mathrm{NaH}_{2} \mathrm{PO}_{4}, 1$ $\mathrm{MgSO}_{4}, 26 \mathrm{NaHCO}_{3}, 10-15$ D-glucose, and $2 \mathrm{CaCl}_{2}$ (bubbled with $5 \%$ $\mathrm{CO}_{2} / 95 \% \mathrm{O}_{2}$ at $32^{\circ} \mathrm{C}$ ). Whole-cell recordings were made from CA3 pyramidal cells or interneurons, with patch electrodes (2-5 M $\Omega$ ) filled with a solution containing the following (in mM): $130 \mathrm{CsMeSO}_{4}, 10 \mathrm{HEPES}$, 0.5 EGTA, 4 Mg-ATP, $0.3 \mathrm{Na}$-GTP, $5 \mathrm{~N}$-(2,6-dimethylphenylcarbamoylmethyl) triethylammonium chloride, and $8 \mathrm{NaCl}$ (285 mOsm), pH 7.2. For recordings of spontaneous network activity, the filling solution was as follows (in mM): $135 \mathrm{~K}$-gluconate, 10 HEPES, 5 EGTA, 4 Mg-ATP, 0.5 $\mathrm{Na}-\mathrm{GTP}, 2 \mathrm{KCl}$, and $2 \mathrm{Ca}(\mathrm{OH})_{2}(285 \mathrm{mOsm}$ ), pH 7.2. LTP230D (www. ltp-program.com) (Anderson and Collingridge, 2001) and WinEDR version 2.3.3 (Strathclyde Electrophysiology Software; University of Strathclyde, Glasgow, UK) software were used for data acquisition. All compounds were from Tocris Cookson (Bristol, UK), except for pertussis toxin, bisindolylmaleimide (BIS), and glutamic-pyruvic transaminase (GPT) (Sigma, St. Louis, MO), and (3S,4aR,6S,8aR)-6-(4-carboxyphenyl) methyl-1,2,3,4,4a,5,6,7,8,8a-decahydroisoquinoline-3-carboxylic acid (LY382884) (Eli Lilly, Indianapolis, IN).

Interneurons were visually identified under infrared illumination combined to differential interference contrast or Dodt gradient optics based on following criteria: (1) localization in the CA3 stratum lucidum and (2) multipolar or bipolar shape of the cell soma, the latter in an orientation perpendicular to the pyramidal cells.

$K A R$-selective drugs. In the present study, we used (RS)-2-amino-3-(3hydroxy-5-tertbutylisoxazol-4-yl)propanoic acid (ATPA) and LY382884 to identify the roles of KARs in the neonatal hippocampus. ATPA is a potent GluR5 subtype-selective agonist (Clarke et al., 1997), which only activates other KAR subtypes and AMPA receptors at higher concentrations than those used in the present study. LY382884 is highly selective for homomeric GluR5 or heteromeric GluR5 subunit-containing KARs (Bortolotto et al., 1999). It has been tested on a variety of other neurotransmitter receptors, including adenosine receptors, $\beta$-adrenoreceptors, and muscarinic receptors, and found to be ineffective (Lauri et al., 2001b), except on AMPA receptors, which it inhibits at concentrations above those used in the present study (Bortolotto et al., 1999). Furthermore, in the present study, we show that the effects of LY382884 on glutamatergic transmission in the neonatal hippocampus were absent in GluR5 ${ }^{-1-}$ mice, strongly suggesting that LY382884 was selectively acting on GluR5 subunit-containing KARs.

Data analysis. Spontaneous activity patterns were analyzed using the Mini Analysis Program, version 5.6.6 (Synaptosoft, Decatur, GA). Spontaneous EPSCs (sEPSCs) and spontaneous IPSCs (sIPSCs) were detected using the peak detection algorithm. The amplitude threshold was set between 4 and $6 \mathrm{pA}$ (two times the baseline rms noise level), and the detected events were verified visually. In experiments in which a lowchloride electrode filling solution was used, network bursts were identified based on a slow outward current, with an amplitude and duration of at least $10 \mathrm{pA}$ and $100 \mathrm{~ms}$, respectively.

The number of spontaneous events was calculated in 120 or $180 \mathrm{~s} \mathrm{bins}$ and normalized to the average baseline value before drug application. In addition, the average number of events per minute was calculated from a 5-10 min period before, during, and after washout of the drug. Neurons that exhibited a very low frequency of miniature EPSCs (mEPSCs) (less than one event every $2 \mathrm{~min} ; ~ 30 \%$ of recorded cells at P4) were excluded from the analysis. Amplitude-frequency distributions were plotted using a bin of $3 \mathrm{pA}$ and normalized by dividing the number of events in each bin by the sum of all events (at least 50 events for each condition). Data are expressed as percentage of control (i.e., $100 \%$ equals no change). All of the pooled data are given as mean \pm SEM for the number of cells indicated. For statistical analysis, Student's two-tailed $t$ test was used, except for the amplitude-frequency distributions, in which Pearson's $\chi^{2}$ test was used. $p<0.05$ was considered statistically significant.

\section{Results}

\section{Pharmacological activation of KARs modulates spontaneous synaptic and network activity in the developing CA3 pyramidal cells}

To study the patterns and regulation of network activity in the developing (P3-P6) hippocampus, we made whole-cell patchclamp recordings from CA3 pyramidal cells in hippocampal slices. To be able to differentiate between GABAergic and glutamatergic activity, the $\mathrm{GABA}_{\mathrm{A}}$ reversal potential was experimentally modified using a low concentration of chloride $(2 \mathrm{mM})$ in the electrode filling solution. When the cells were voltage clamped at $-62 \mathrm{mV}$ under these conditions, the $\mathrm{GABA}_{\mathrm{A}}$ receptor-mediated synaptic events (IPSCs) were observed as outward currents, and glutamatergic synaptic events (EPSCs) were observed as inward currents (Fig. 1A). Spontaneous activity in the immature hippocampus has a typical pattern of network-driven bursts (average frequency, $3.4 \pm 0.4$ events/min; $n=17$ ) that are interspersed with occasional isolated glutamatergic events (sEPSCs; $5.5 \pm 1.2$ events/min; $n=12$ ) and more frequent GABAergic synaptic events (sIPSCs; $42.4 \pm 5.3$ events/min; $n=15$ ). As described previously (Khazipov et al., 1997; Lamsa et al., 2000), the network bursts consist of a large, long-lasting outward GABAergic current with a barrage of fast inward EPSCs superimposed on it (Fig. $1 A$ ).

To find out whether KARs containing the GluR5 subunit can regulate this spontaneous activity, we first tested the effect of the GluR5-selective KAR agonist ATPA (Clarke et al., 1997). Bath application of ATPA $(1 \mu \mathrm{M})$ caused a substantial and reversible block of the network bursts (18.5 $\pm 7 \%$ of control) (Fig. 1B,C). This effect was associated with an increase in the frequency $(392 \pm 89 \%$ of control; $p<0.005$ ) (Fig. $1 D$ ) but no change in the amplitude $(92 \pm 7 \%)$ or kinetics (rise and decay times, $92 \pm 6$ and $103 \pm 1 \%$, respectively) of sIPSCs $(n=6)$. ATPA also caused a significant inhibition in the frequency $(43 \pm 10 \% ; p<0.05)$ (Fig. $1 E$ ) but no change in the amplitude $(97 \pm 4 \%)$ or kinetics (rise and decay times, $123 \pm 18$ and $103 \pm 4 \%$, respectively) of sEPSCs $(n=5)$. To test whether ATPA was selectively affecting a 
A i)

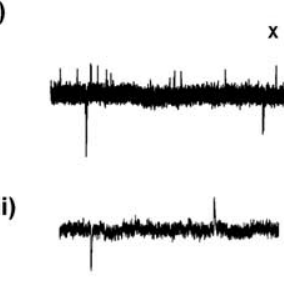

ii)
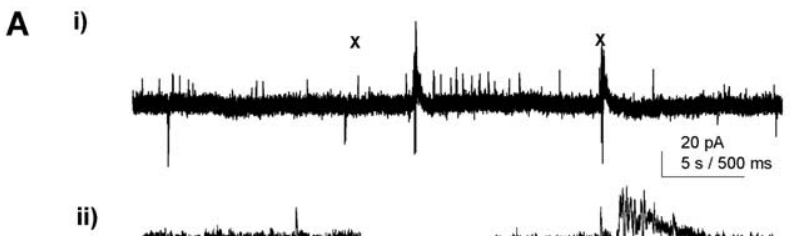

iii)

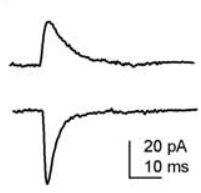

B

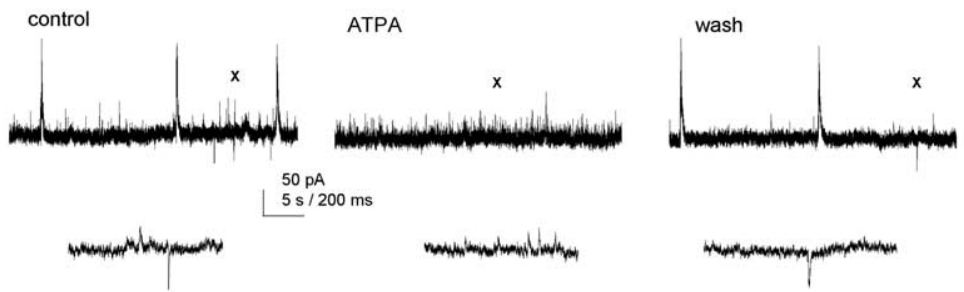

C network bursts
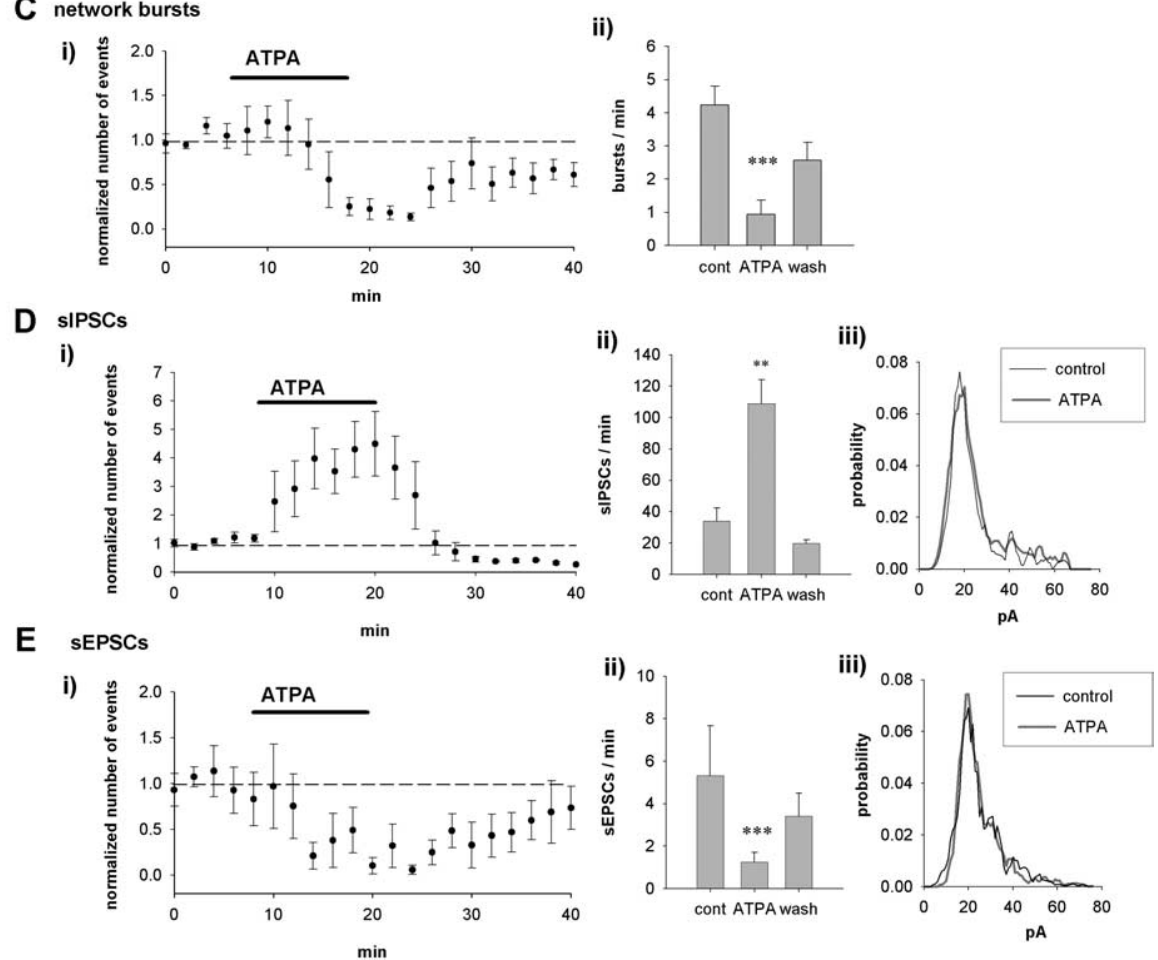

Figure 1. Network and synaptic activity in the neonatal hippocampus is regulated by KAR activation. $A$, A typical pattern of spontaneous activity under control conditions, recorded from a CA3 pyramidal neuron (P4), using a low-chloride solution in the patch electrode (Ai). sIPSCs are seen as outward currents, and sEPSCs are seen as inward currents, as shown in an expanded time scale (Aii, left). The network bursts consist of a slow GABAergic current and a barrage of EPSCs (Aii, right). In all figures, X marks the place of the expanded time-scale trace. Aiii, An average of 10 sIPSCS (top) and sEPSCs on an expanded scale. $\boldsymbol{B}$, Example traces showing that ATPA $(1 \mu \mathrm{m})$ inhibits network bursts and the frequency of sEPSCs and increases the frequency of sIPSCs. C, Pooled data $(n=5)$ showing the effect of ATPA on the frequency of network bursts. $C i$, The number of spontaneous events as a function of time, calculated in 120 s bins and normalized to the baseline level before application of ATPA. Cii, The average number of events per minute before, in the presence of, and after washout (wash) of ATPA. cont, Control. $\boldsymbol{D}$, Equivalent data from the same neurons for sIPSCS. Diii, The amplitude-frequency distribution of sIPSCs under control conditions and in the presence of ATPA.E, Equivalent data from the same neurons for sEPSCS. Error bars represent SEM. ${ }^{* *} p<0.005$ and ${ }^{* *} p<0.01$ compared with control.

subpopulation of spontaneous events, we analyzed its effect on the amplitude distribution of sIPSCs and sEPSCs. There were no significant changes in the amplitude distribution of either sIPSCs ( $p=0.26$ ) (Fig. 1 Diii) or sEPSCs $(p=0.26)$ (Fig. 1 Eiii) in the presence of ATPA. Thus, pharmacological activation of KARs can dramatically affect synaptic transmission in CA3 pyramidal neurons by increasing sIPSC frequency, decreasing sEPSC frequency, and inhibiting synchronized network activity.
Endogenous activation of KARs regulates spontaneous EPSCs, but not IPSCs, in the CA3 pyramidal cells

To understand the physiological role of KARs in this network activity, we tested the effect of the GluR5-selective KAR antagonist LY382884 (Bortolotto et al., 1999; Lauri et al., 2001a) on spontaneous activity. LY382884 $(10 \mu \mathrm{M})$ had no effect on the input resistance or holding current of the pyramidal neurons but decreased the frequency of network bursts ( $72 \pm 7 \%$; $p<$ $0.005 ; n=12$ ) (Fig. 2A,B). LY382884 had no effect on the frequency $(95 \pm 12 \%)$ (Fig. $2 A, C$ ), the amplitude ( $98 \pm 6 \%$ ), or the amplitude distribution $(p=0.22)$ (Fig. 2Ciii) of sIPSCs $(n=9)$. However, LY382884 dramatically and reversibly increased the frequency of sEPSCs $(261 \pm$ $28 \%$; $p<0.01$ ) (Fig. $2 A, D$ ) without affecting the amplitude ( $94 \pm 4 \%)$, the kinetics (rise and decay times, $103 \pm 9$ and $98 \pm$ $4 \%$, respectively; $n=12$ ), or the amplitude distribution ( $p=0.24)$ (Fig. 2Diii).

To ensure that these effects were specifically mediated by LY382884 acting on GluR5 subunit-containing KARs, we next tested the antagonist on genetically modified mice lacking GluR5 (Mulle et al., 2000). In the wild-type mice (P3-P6), LY382884 caused a reversible increase in sEPSC frequency in CA3 pyramidal cells $(145 \pm 5 \% ; p<0.005 ; n=6)$ (Fig. $2 E$ ). However, in GluR5 ${ }^{-1-}$ mice, LY382884 had no effect on sEPSCs $(96 \pm 7 \% ; n=5$ ) (Fig. 2E). These data show, therefore, that GluR5 subunit-containing KARs are tonically activated in the neonatal hippocampus by endogenous L-glutamate and that this activation preferentially downregulates glutamatergic input to $\mathrm{CA} 3$ pyramidal cells.

The role of KARs in the regulation of spontaneous interneuronal activity in the developing hippocampus

Network bursting in the immature hippocampus is critically dependent on synchronous activity in the interneuronal network (Khazipov et al., 1997; Lamsa et al., 2000). To further understand the regulation of the developing network activity by KARs, we next recorded spontaneous neuronal activity from visually identified interneurons in the CA3 stratum lucidum. Compared with pyramidal neurons, the pattern of the spontaneous activity in interneurons was characterized by a much higher frequency of sEPSCs $(120 \pm 24$ events/min, $n=19$; in pyramidal cells, $5.5 \pm 1.2$ events $/ \mathrm{min}, n=12 ; p<0.001)$, whereas the occurrence of sIPSCs ( $49 \pm 9$ events/min; $n=13$ ) and network bursts $(4.9 \pm 1 ; n=13)$ was similar to that in the pyramidal neurons. In addition, the amplitude distribution of sEPSCs in interneurons was wider than in pyramidal neurons $(p<0.005)$ 
(Figs. $1 E, 2 D$ for pyramidal neurons vs Figs. 3E, $4 E$ for interneurons), suggesting variability in their synaptic origin.

Similar to pyramidal cells, bath application of the KAR agonist ATPA caused a robust inhibition of the network bursts in interneurons ( $44 \pm 16 \%$; $p<0.005$ ) (Fig. $3 A, B)$. The effect of ATPA was associated with an increase in the frequency (304 \pm $42 \% ; p<0.005 ; n=11$ ) (Fig. $3 C$ ) but no change in the amplitude $(117 \pm 11 \%)$ or kinetics (rise time, $108 \pm 3 \%$; decay time, $113 \pm 13 \%$ ) of sIPSCs. Interestingly, however, in interneurons, ATPA had a biphasic effect on sEPSC frequency. Application of ATPA resulted in a transient increase in sEPSC frequency $(147 \pm 14 \% ; p<0.05)$, which was followed by depression $(75 \pm$ $8 \% ; p=0.09$ ) (Fig. 3D). ATPA had no effect on sEPSC amplitude (104 $\pm 4 \%$ ) or kinetics (rise and decay times, $106 \pm 7$ and $105 \pm 3 \%$ of control, respectively). Analysis of the amplitude distribution did not reveal any significant changes in sIPSCs $(p=0.23)$ or sEPSCs $(p=0.21)$ (Fig. $3 E)$. Thus, although the network bursting and the frequency of sIPSCs were similarly regulated in CA3 pyramidal neurons and interneurons, pharmacological activation of KARs had opposite effects on spontaneous glutamatergic transmission in these cell types.

To study whether endogenously activated KARs also contribute to the regulation of spontaneous synaptic activity in the interneurons, we tested the effects of LY382884. Application of LY382884 resulted in a decrease in the frequency of network bursts (burst frequency, $66 \pm 8 \%$ of control; $n=10$; $p<0.05$ ) (Fig. $4 A, B$ ) but had no effect on the frequency ( $99 \pm 8 \%$ ) or amplitude $(104 \pm 5 \%)$ of sIPSCs $(n=$ 10) (Fig. 4C). However, LY382884 significantly reduced the frequency $(69 \pm 6 \%$; $p<0.01 ; n=10$ ) of sEPSCs but had no effect on their amplitude (99 $\pm 3 \%$ ), kinetics (rise time, $108 \pm 5 \%$; decay time, $99 \pm 1 \%$ of control), or amplitude distribution $(p=0.19)$ (Fig. $4 D, E)$. These data show that, in contrast to pyramidal cells, endogenous activation of KARs facilitates glutamatergic input onto interneurons.

\section{Activation of KARs differentially regulates the frequency of mEPSCs at CA3 pyramidal neurons and interneurons}

The lack of effect of both ATPA and LY382884 on the sEPSC amplitude or kinetics suggests that their effects on spontaneous AMPA receptor-mediated transmission are attributable to a presynaptic mechanism. Both direct (i.e., action potential independent) and indirect (e.g., regulation of interneuron firing frequency and change in axon excitability) mechanisms for KARs in regulating transmitter release have been described previously (Kamiya and Ozawa, 2000; Cossart et al., 2001; Frerking et al.,

A
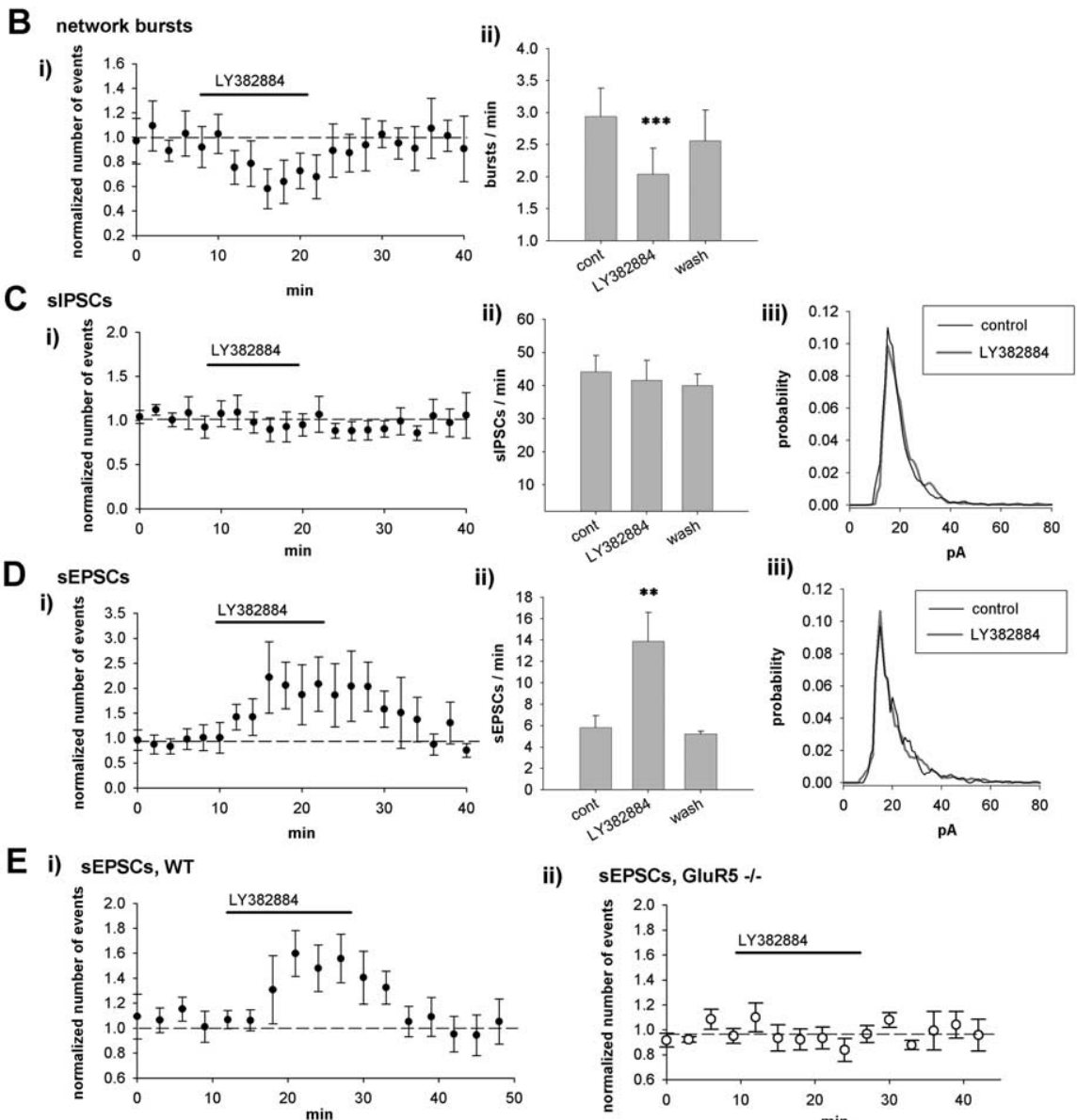

ii) SEPSCs, GluR5 \%

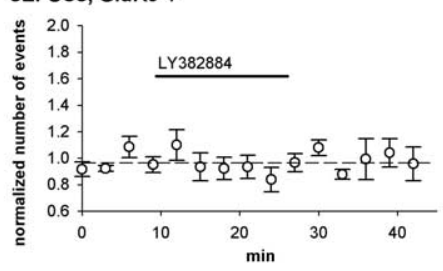

Figure 2. Endogenously activated KARs regulate spontaneous activity in the neonatal hippocampus. $\boldsymbol{A}$, Example recordings from a CA3 pyramidal neuron (P4) showing the effect of LY382884 (10 $\mu \mathrm{M})$ on spontaneous network activity. LY382884 reduces the occurrence of network bursts, increases the frequency of sEPSCS, but has no effect on sIPSCS. B, Pooled data $(n=12)$, presented as in Figure 1, showing the effects of LY382884 on spontaneous network bursts. C, Equivalent data from the same Y382884. D, Equivalent data from the same neurons for sEPSCS. $\boldsymbol{E}$, Pooled data of recordings of spontaneous isolated EPSCs from wild-type (WT; $n=6)$ and GluR5 ${ }^{-1-}(n=5)$ mice (P3-P6), showing that the effect of LY382884 on glutamatergic transmission is dependent on GluR5. wash, Washout. Error bars represent SEM. ${ }^{* * *} p<0.005$ and ${ }^{* *} p<0.01$ compared with control.

2001; Schmitz et al., 2001; Semyanov and Kullmann, 2001). To test between these possibilities, we next recorded action potential-independent EPSCs (mEPSCs) in the presence of TTX $(1 \mu \mathrm{M})$ and blockers of $\mathrm{GABA}_{\mathrm{A}}, \mathrm{GABA}_{\mathrm{B}}$, and NMDA receptors [100 $\mu \mathrm{m}$ picrotoxin, $1 \mu \mathrm{M}(2 S)-3[[(1 S)$-1-(3,4-dichlorophenyl)ethyl]amino-2-hydroxypropyl](phenylmethyl)phosphinic acid (CGP55845A), and $50 \mu \mathrm{M}$ D-AP-5, respectively].

In immature CA3 pyramidal neurons, LY382884 reversibly increased the frequency of mEPSCs $(186 \pm 22 \% ; p<0.005 ; n=$ 8) (Fig. $5 A, C$ ) without affecting the amplitude (92 $\pm 10 \%)$. ATPA caused a reduction in the frequency of mEPSCs (70 $\pm 7 \%$; $p<0.005 ; n=9$ ) (Fig. 5D) but no change in the amplitude (98 \pm $4 \%)$. The amplitude distribution of mEPSCs in pyramidal cells 
A

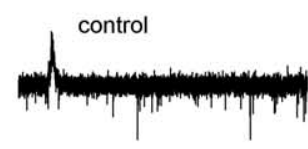

B network bursts

i )

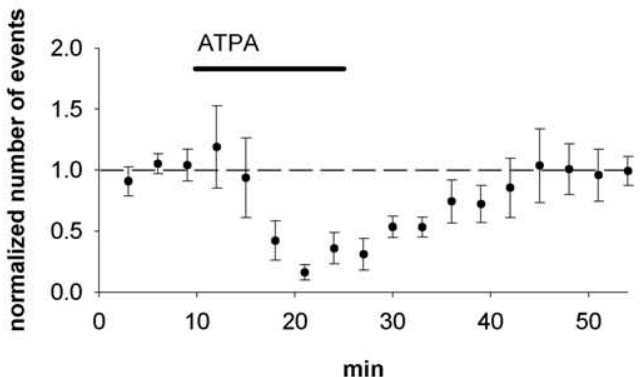

C sIPSCs

i )

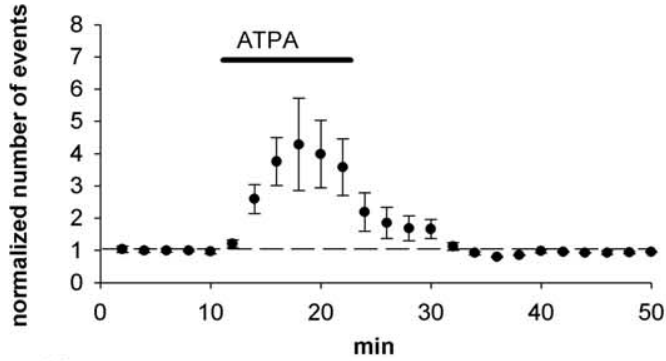

D sEPSCs

i)

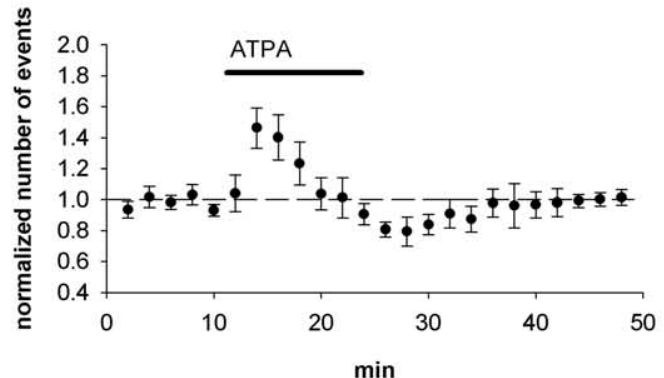

E i) sIPSCs

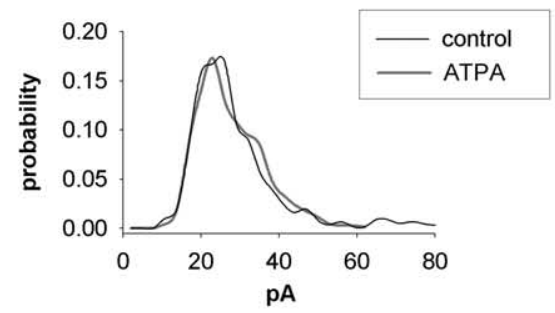

ii )

ii) SEPSCs

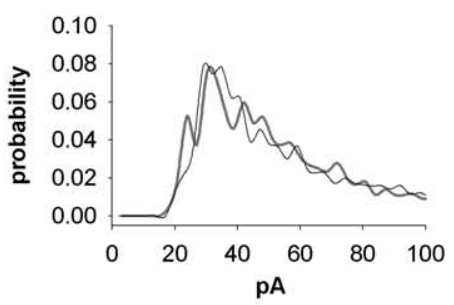

Figure 3. KAR activation regulates spontaneous activity in CA3 interneurons. $A$, Example recordings from a $C A 3$ stratum lucidum interneuron (P4) showing the effect of ATPA $(1 \mu \mathrm{M})$ on spontaneous network activity. ATPA inhibits network bursts but increases the frequency of sEPSCS and SIPSCS. $B$, Pooled data $(n=11)$ showing the effects of ATPA on spontaneous network bursts. C, Equivalent data from the same neurons for sIPSCS. D, Equivalent data from the same neurons for sEPSCS. $E$, Amplitude distribution of sIPSCS (EI) and sEPSCS (Eii) under control conditions and in the presence of ATPA. wash, Washout. Error bars represent SEM. ${ }^{* *} p<0.01$ and ${ }^{*} p<0.05$ compared with control.

was not altered by ATPA $(p=0.33)$ or LY382884 $(p=0.36)$ (Fig. $5 E$ ). Thus, tonically active KARs act to depress glutamate release in an action potential-independent manner at the glutamatergic synapses onto CA3 pyramidal cells in the neonatal hippocampus.

In CA3 interneurons, however, LY382884 reversibly decreased the frequency of mEPSCs $(62 \pm 8 \% ; n=8 ; p<0.05)$ but had no effect on their amplitude $(97 \pm 6 \% ; n=8)$. KAR activa- tion by ATPA increased the frequency of mEPSCs $(163 \pm 21 \% ; p<0.05 ; n=10)$ (Fig. 5) without affecting their amplitude $(98 \pm 4 \%)$. The initial facilitation of mEPSC frequency after application of ATPA was followed by depression (44 \pm $8 \% ; n=10 ; p<0.005)$, suggesting two opposite effects of KAR activation on glutamate release at these synapses. Therefore, during early development, endogenously activated KARs preferentially depress release at glutamatergic terminals onto CA3 interneurons. In the amplitude distributions, there was a trend toward a shift to high-amplitude mEPSCs ( $p=$ 0.058) in the presence of ATPA and, correspondingly, a trend toward a shift to lowamplitude mEPSCs in the presence of the KAR antagonist LY382884 ( $p=0.061$ ) (Fig. $5 E$ ). This could suggest that KARs differentially regulate subpopulations of spontaneous events in interneurons. At P14-P16, LY382884 had no effect on the frequency or amplitude of mEPSCs in either pyramidal neurons ( $93 \pm 9$ and $103 \pm 6 \%$, respectively; $n=8)$ or interneurons ( $89 \pm 11$ and $102 \pm$ $7 \% ; n=6$ ) (Fig. $5 F$ ), indicating that these effects are developmentally regulated.

In summary, these data show that glutamatergic terminals in area CA3 of the neonatal, but not 2-week-old, hippocampus express KARs that are activated by endogenous L-glutamate and act to regulate L-glutamate release in an action potentialindependent manner. These receptors inhibit glutamate release at synapses onto pyramidal neurons and facilitate release at glutamatergic synapses onto interneurons.

\section{LY382884-sensitive KARs are not tonically activated on interneurons in the neonatal hippocampus}

This direct regulation of glutamate release at terminals by KARs can fully explain the observed effects of LY382884 on spontaneous EPSCs both in pyramidal cells and in interneurons. However, additional mechanisms, such as activation of somatodendritic KARs on interneurons, might contribute to the effect of LY382884 on the network bursts. To find out whether somatodendritic KARs on interneurons are tonically active, we analyzed the effects of ATPA and LY382884 on the holding current of interneurons from the experiments shown in Figures 3 and 4. LY382884 had on average no effect on the holding current of interneurons ( $6 \pm 4 \mathrm{pA} ; n=7)$; however, under similar conditions, ATPA caused a significant inward current $(79 \pm 9$ pA; $p<0.01 ; n=10)$ (Fig. $6 A$ ). Thus, LY382884sensitive somatodendritic KARs do not mediate a sustained inward current in interneurons, a finding consistent with the lack of effect of LY382884 on sIPSCs in either pyramidal neurons or interneurons. 
KARs have been shown to regulate action potential-independent GABA release in adult CA1 (Rodriguez-Moreno and Lerma, 1998; Mulle et al., 2000; Cossart et al., 2001) (but see Frerking et al., 1999; Jiang et al., 2001; Semyanov and Kullmann, 2001). To study whether KARs influence GABA release in the neonatal hippocampus by a direct action at GABAergic terminals, we next recorded miniature IPSCs (mIPSCs) from CA3 pyramidal neurons and interneurons in 3- to 6-d-old rats in the presence of $1 \mu \mathrm{M}$ TTX, $1 \mu \mathrm{M}$ CGP55845A, $50 \mu \mathrm{M} 1-$ (4-aminophenyl)-4-methyl-7,8-methylenedioxy-5H-2,3-benzodiazepine (GYKI53655), and $50 \mu \mathrm{M}$ D-AP-5. LY382884 had no effect on the frequency or amplitude (107 \pm 4 and $100 \pm 2 \%$, respectively; $n=4$ ) of mIPSCs in CA3 pyramidal neurons or interneurons (frequency, $98 \pm 10 \%$; amplitude, $97 \pm 2 \%$; $n=6$ ) (Fig. $6 B, D$ ). Moreover, bath application of ATPA had no effect on mIPSCs, recorded from neonatal CA3 pyramidal neurons (frequency, $112 \pm$ $15 \%$; amplitude, $99 \pm 5 \%$; $n=4$ ) or interneurons (frequency, $101 \pm 10 \%$; amplitude, $96 \pm 5 \%$; $n=11$ ) (Fig. $6 C, D$ ). Furthermore, there were no significant changes in the amplitude distributions of mIPSCs (data not shown). Thus, GluR5-containing KARs do not regulate action potential-independent GABA release in neonatal CA3.

Inhibitory, but not facilitatory, effects of KARs on glutamate release are dependent on pertussis toxin-sensitive G-proteins and PKC

The depression of excitatory synaptic transmission in area CA1 by the pharmacological activation of KARs (Chittajallu et al., 1996; Frerking et al., 2001; Clarke and Collingridge, 2002) has been suggested to involve a G-protein-mediated mechanism (Frerking et al., 2001), similar to that involved in the regulation of synaptic inhibition (Rodriguez-Moreno and Lerma, 1998). Thus we next explored whether the effects of KARs on glutamate release in the neonatal hippocampus are dependent on PTX-sensitive G-proteins. Slices (P3-P6) were maintained overnight with or without PTX $(5 \mu \mathrm{g} / \mathrm{ml})$. This treatment with PTX fully blocked the depression of IPSCs in response to application of the $\mathrm{GABA}_{\mathrm{B}}$ receptor agonist baclofen (Fig. 7A), indicating that PTX was effective in blocking G-protein-mediated signaling under these conditions. In CA3 pyramidal neurons, the changes in mEPSC frequency after pharmacological manipulation of GluR5 KARs in slices incubated overnight under control conditions were of a magnitude similar to that observed in acute slices (ATPA, $43 \pm 7 \%$ of control, $n=7$, $p<0.05$; LY382884, $177 \pm 10 \%, n=7, p<0.005)$ (Fig. $7 B$ ). In PTX-treated slices, however, ATPA and LY382884 had no effect on $\mathrm{mEPSC}$ frequency (Fig. $7 \mathrm{~B}$ ), although a significant increase in

A

B network bursts
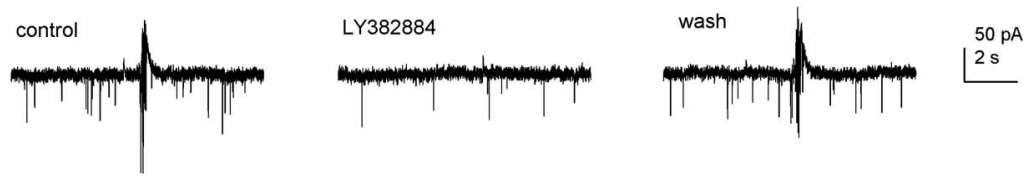

i)

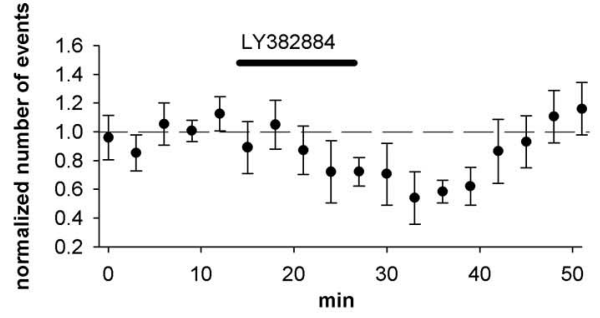

C sIPSCs

i)

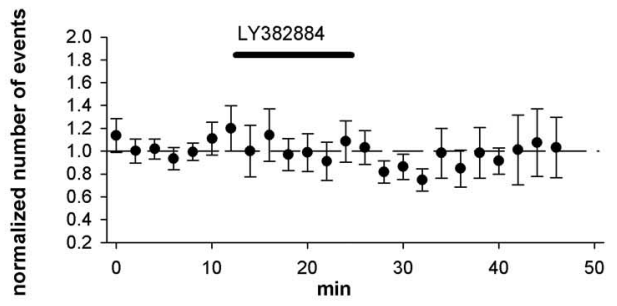

sEPSCs

i)

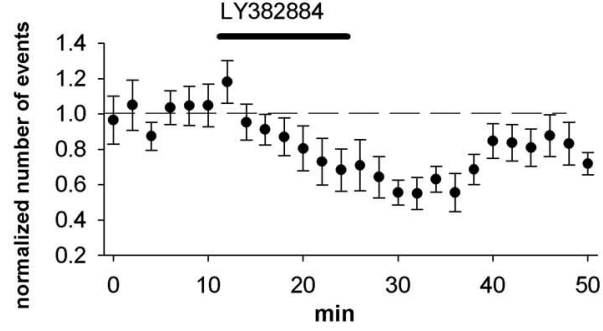

E

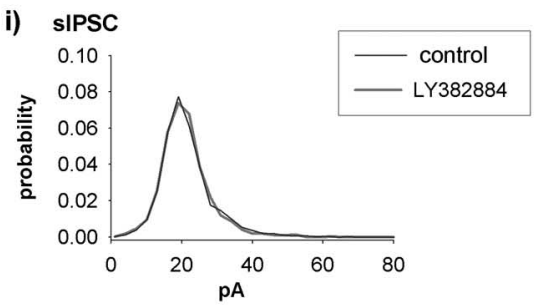

ii)

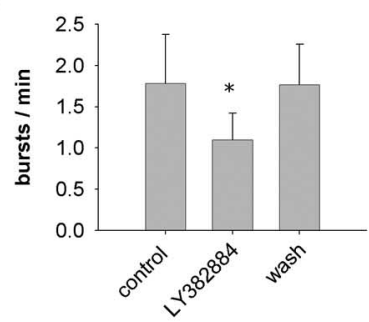

ii)

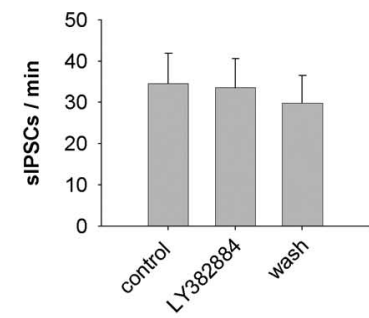

ii)

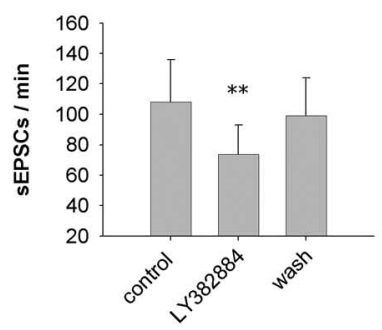

ii) SEPSC

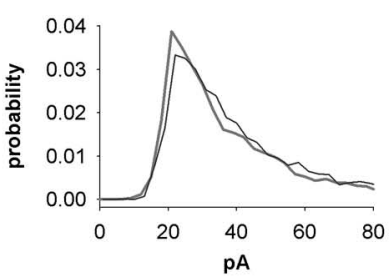

Figure 4. Effect of $L Y 382884$ on spontaneous activity in $C A 3$ interneurons. $A$, Example recordings from a $C A 3$ stratum lucidum interneuron (P4) showing the effect of LY382884 (10 $\mu \mathrm{M})$ on spontaneous network activity. LY382884 attenuates the occurrence of network bursts and sEPSCs but has no effect on sIPSCs. B, Pooled data $(n=10)$, presented as in Figure 1, showing the effects of LY382884 on spontaneous network bursts. C, Equivalent data from the same neurons for sIPSCS. D, Equivalent data from the same neurons for SEPSCS. E, Amplitude distribution of SIPSCS (EI) and SEPSCS (Eii) under control conditions and in the presence of LY382884. wash, Washout. Error bars represent SEM. ${ }^{* *} p<0.01$ and ${ }^{*} p<0.05$ compared with control.

the frequency could still be induced with $4 \mathrm{~mm} \mathrm{KCl} \mathrm{(mEPSC}$ frequency, $156 \pm 10 \% ; n=5 ; p<0.05$; data not shown).

In contrast, the effect of KAR activation or inhibition on mEPSC frequency at interneurons was not blocked after PTX pretreatment. In PTX-pretreated slices, ATPA was still able to facilitate mEPSC frequency in interneurons (148 $\pm 10 \% ; n=8$; $p<0.01)$, in a manner similar to that in slices incubated in control conditions $(151 \pm 15 \% ; n=8 ; p<0.05)$ (Fig. 7C). Also, LY382884 caused reduction in $\mathrm{mEPSC}$ frequency in interneurons of PTX incubated slices ( $56 \pm 7 \% ; n=9 ; p<0.05$ ), in a manner 
A pyramidal cell

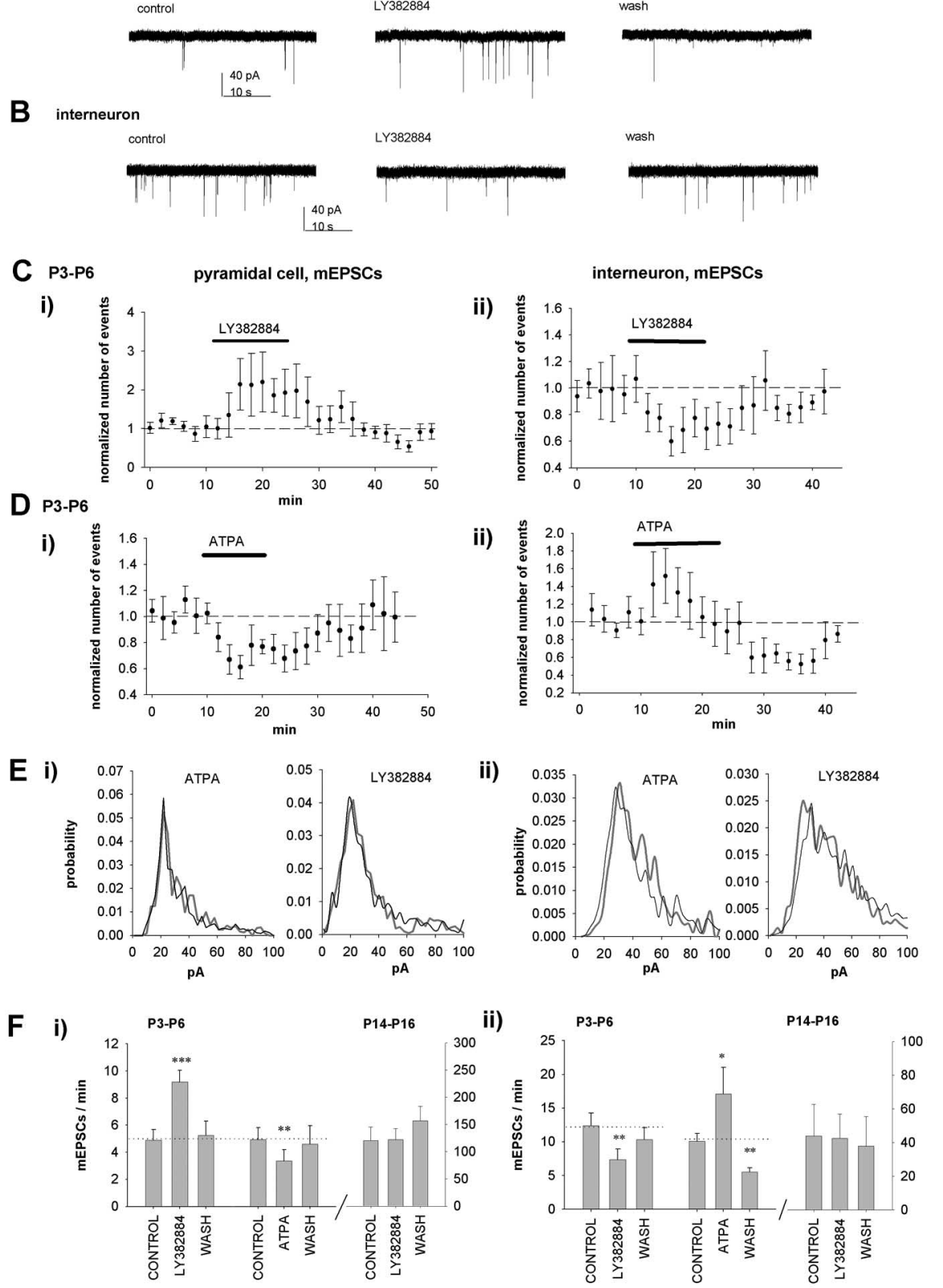

Figure 5. KARs regulate mEPSC frequency in a differential manner in CA3 pyramidal neurons and interneurons in the neonatal, but not 2-week-old, rat hippocampus. $A$, Example traces showing that LY382884 causes a reversible increase in the frequency of $m E P S C s$ in a CA3 pyramidal neuron at P4. $B$, Example traces showing that $L Y 382884$ causes a reversible decrease in the frequency of mEPSCs in a CA3 stratum lucidum interneuron at P4. C, Pooled data on the effect of LY382884 on mEPSC frequency in CA3 pyramidal neurons ( $C$ i $)(n=8)$ and interneurons ( $C i i)(n=10)$. The number of $m E P S C s$ is calculated in 120 s bins and normalized to the baseline level before application of $L Y 382884$. D, Equivalent data for the effect of ATPA on mEPSCs in pyramidal neurons (Di) $(n=8)$ and interneurons (Dii) $(n=10)$ at P3-P6. E, Normalized amplitude distribution of mEPSCs under control conditions (black trace) or in the presence of ATPA or LY382884 (gray trace) in the P4-P6 pyramidal neurons (Ei) and interneurons (Eii). $\boldsymbol{F}$, Pooled data presenting the average number of mEPSCs per minute before, during, and after pharmacological manipulation of KARs in neonatal and 2-week-old CA3 pyramidal neurons (Fi) and interneurons (Fii). wash, Washout. Error bars represent SEM. ${ }^{* * *} p<0.005,{ }^{* *} p<0.01$, and ${ }^{*} p<0.05$ compared with control.

similar to that in control incubated slices $(58 \pm 6 \% ; n=7 ; p<$ 0.05) (Fig. 7C). Interestingly, however, in PTX-treated slices, the ATPA-induced initial facilitation of mEPSC frequency was not followed by depression ( $99 \pm 18 \% ; p=0.5)$, although in control slices, a significant depression of mEPSC frequency $(74 \pm 15 \%$; $p<0.05$ ) was seen $15-20 \mathrm{~min}$ after application of ATPA (Fig.
7C). Thus, the presynaptic KARs inhibiting glutamate release in neonatal CA3 act via a G-protein-mediated mechanism, whereas the facilitatory effect is not dependent on PTX-sensitive G-proteins.

The KARs acting via a G-proteinmediated signaling mechanism have been shown to couple to activation of PKC (Rodriguez-Moreno and Lerma, 1998). Therefore, we next tested whether the inhibitory and facilitatory effects of KAR in the neonatal hippocampus are dependent on PKC activation. The hippocampal slices were preincubated for at least $30 \mathrm{~min}$ in the presence of bisindolylmaleimide VIII acetate $(0.5 \mu \mathrm{M})$, a selective inhibitor for PKC (Toullec et al., 1991), after which the effect of KAR-selective pharmacological agents on mEPSC frequency was tested. In pyramidal cells, in the presence of BIS, neither ATPA or LY382884 had an effect on mEPSC frequency (ATPA, $101 \pm 10 \%$ of control, $n=8, p=0.58$; LY382884, $114 \pm 7 \%$ of control, $n=7, p=0.36$ ) (Fig. $7 D)$. In interneurons, however, in the presence of BIS, ATPA application still resulted in transient facilitation of mEPSC frequency $(137 \pm 10 \% ; n=9 ; p<0.005)$, but, in contrast to control conditions, no depression was observed $(100 \pm 18 \%$; $p=$ 0.36) (Fig. 7E). In interneurons, LY382884 inhibited mEPSC frequency in the presence of BIS ( $63 \pm 5 \% ; n=6$; $p<0.05$ ) (Fig. $7 E$ ) in a manner similar to control. These data show that BIS and PTX have identical effects on the regulation of glutamate release by KARs. Thus, the inhibitory, but not facilitatory, KARs regulating glutamate release in the neonatal hippocampus are coupled to intracellular signaling mechanisms involving pertussis toxin-sensitive G-proteins and PKC.

\section{Ambient levels of L-glutamate regulate presynaptic KARs in the neonatal hippocampus}

One possible mechanism to explain the tonic activation of presynaptic glutamate receptors in neonatal, but not in 2-week-old, CA3 is differences in the extracellular concentrations and/or diffusion of glutamate attributable to developmental changes in the glutamate transport mechanisms and/or the composition of the extracellular space ( $\mathrm{Ru}$ sakov and Kullmann, 1998; Sykova et al., 2000; Danbolt, 2001). To test whether decreasing the extracellular glutamate concentration regulates $\mathrm{MEPSC}$ frequency and its regulation by the activation of presynaptic KARs, we used an enzymatic "glutamate scavenger" [GPT plus pyruvate (2 mM)] (Overstreet et al., 1997; Min et al., 1998). Because it has been reported that manipulations of L-glutamate uptake can enable the activation of presynaptic metabotropic Glu (mGlu) receptors (Scanziani et al., 1997), we added a 
broad-spectrum mGlu receptor antagonist, 2S-2-amino-2-(1S,2S-2-carboxycyclopropyl-1-yl)-3-(xanth-9-yl)propanoic acid (LY341495), at a concentration $(100 \mu \mathrm{M})$ that blocks the activation of mGluR1mGluR8 (Fitzjohn et al., 1998). Treatment with LY341495 had no effect on the ability of LY382884 to increase mEPSC frequency under control conditions (with $100 \mu \mathrm{M}$ LY341495, $178 \pm 19 \%, n=8, p<0.01$;

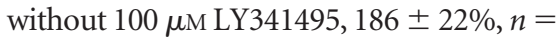
$9, p<0.005$; data not shown).

We found that glutamate release probability, assessed by the frequency of mEP$\mathrm{SCs}$, is highly sensitive to manipulations affecting extracellular glutamate concentration in neonatal, but not 2 -week-old, CA3 (Fig. 8). Thus, the glutamate scavenger caused a robust increase in the mEPSC frequency at P3-P6 (172 $\pm 14 \% ; n=12$; $p<0.005$ ) (Fig. 8A). GPT alone (95 \pm $12 \% ; n=6 ; p=0.81$ ) or pyruvate alone $(97 \pm 7 \% ; n=4 ; p=0.19)$ had no effect on mEPSC frequency (Fig. $8 C$ ). Interestingly, after application of the scavenger, LY382884 had no additional effect on mEPSC frequency at P3-P6 (98 $\pm 8 \%$; $n=$ 12) (Fig. $8 A, C$ ). This was not attributable to a saturation of mEPSC frequency to a ceiling point, because $4 \mathrm{~mm} \mathrm{KCl}$ could still induce an additional increase in mEPSC frequency in the presence of the scavenger (264 $\pm 32 \% ; n=7 ; p<0.05$ compared with scavenger alone). We also tested the effects of blocking glutamate transport on mEPSC frequency. Consistent with this manipulation, raising ambient levels of L-glutamate, the transport inhibitor DLthreo- $\beta$-benzyloxyaspartic acid $(50 \mu \mathrm{M})$, caused a reversible depression of mEPSC frequency at P3-P6 (59 $\pm 8 \% ; n=6 ; p<$ $0.05)$; however, it was without effect at $\mathrm{P} 14-\mathrm{P} 16$ (108 $\pm 25 \% ; n=7 ; p=0.5$ ) (Fig. $8 B, C)$. Thus, endogenous L-glutamate provides a tonic, but submaximal, activation of presynaptic KARs at CA3 terminals in the neonatal hippocampus.

\section{Discussion}

We here identified several novel features of the physiological functions of KARs. We show that (1) KARs can control the activity patterns of the developing neuronal network by regulating the balance between GABAergic and glutamatergic transmission; (2) KARs can differentially regulate glutamate release via an action potentialindependent mechanism at CA3 pyramidal neurons and interneurons; (3) different signaling mechanisms downstream from the KAR activation are used for the facilitatory and inhibitory actions on glutamate release; (4) neonatal KARs are tonically activated by endogenous L-glutamate present in the extracellular space; and (5) this activation mechanism of KARs is developmentally downregulated.
A i)

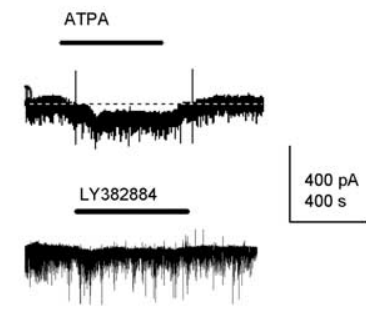

B i)

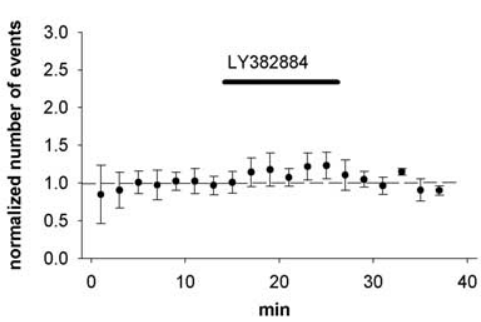

C i)

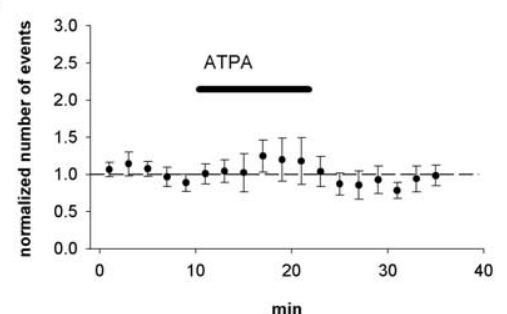

D i)

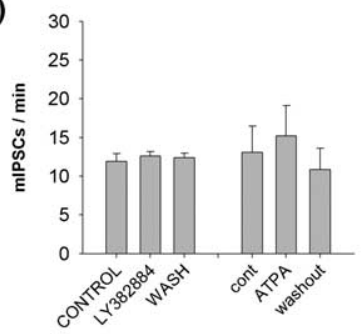

ii)

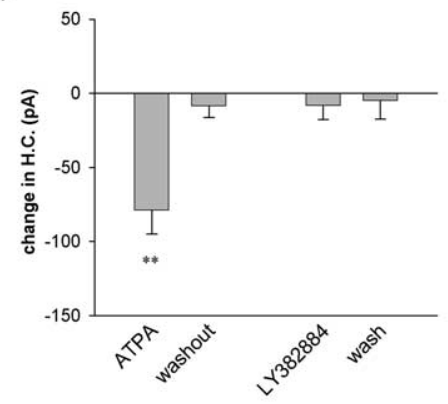

ii

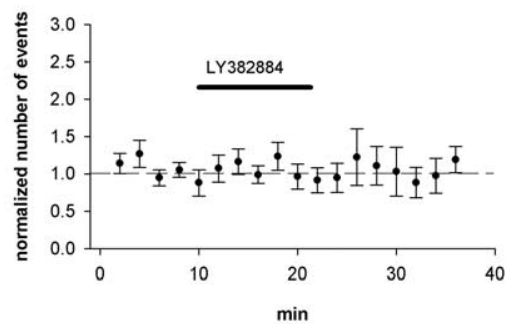

ii)

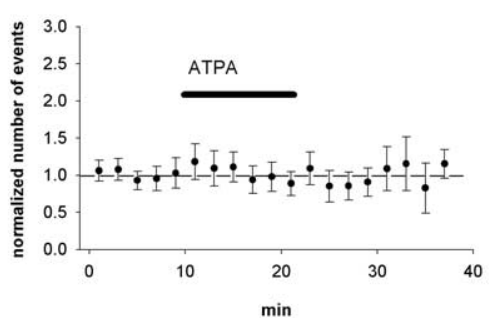

ii )

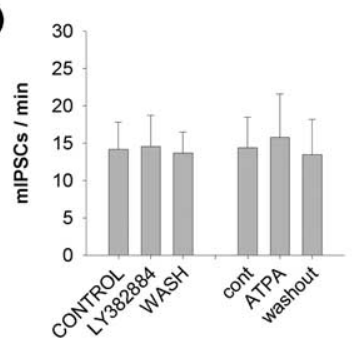

Figure 6. KARs are not tonically activated at interneurons in the neonatal CA3.A, Effect of ATPA $(n=10)$ and LY382884 $(n=$ 7) on holding current of interneurons. Example traces (Ai) and pooled data (Aii) show activation of an inward current in the interneurons in the presence of ATPA but not LY382884. ${ }^{* *} p<0.01$ compared with control. $\boldsymbol{B}$, Pooled data of the effect of LY382884 on mIPSCs at CA3 pyramidal neurons $(B i)(n=4)$ and interneurons ( $B$ ii) $(n=6)$ at P3-P6. The number of mIPSCs has been calculated in $120 \mathrm{~s}$ bins and normalized to the baseline level before application of LY382884. C, Equivalent data on the effect of ATPA on mIPSC frequency at pyramidal neurons $(n=4)$ and interneurons $(n=11)$. $D$, Summary data presenting the lack of effect of LY382884 and ATPA on the frequency of mIPSCs at neonatal CA3 pyramidal neurons (Di) and interneurons (Dii). The values represent the average number of mIPSCS per minute before, during, and after pharmacological manipulation of KARs. wash, Washout; cont, control. Error bars represent SEM.

Regulation of early hippocampal network activity by KARs Rhythmically patterned spontaneous network activity is an inherent property of the developing hippocampus seen both in vitro and in vivo (Ben-Ari et al., 1989; Garaschuk et al., 1998; Palva et al., 2000; Lahtinen et al., 2002; Leinekugel et al., 2002) and is thought to be instrumental in the development of hippocampal circuitries (Luthi et al., 2001; Groc et al., 2002; Lauri et al., 2003b). The initiation and synchronization of the activity are dependent on both GABA and glutamatergic activity in a 

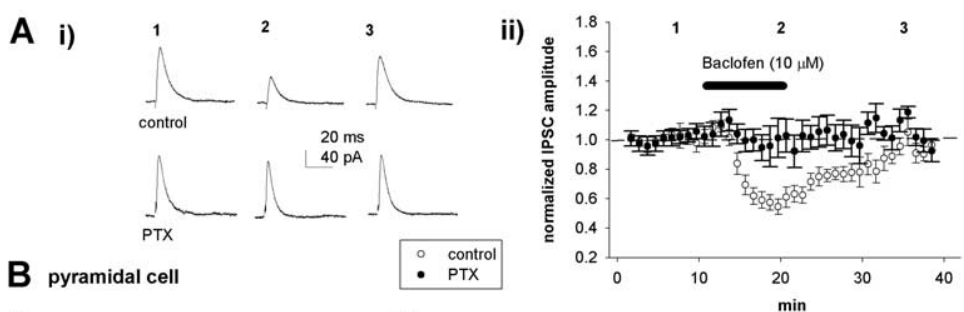

i)

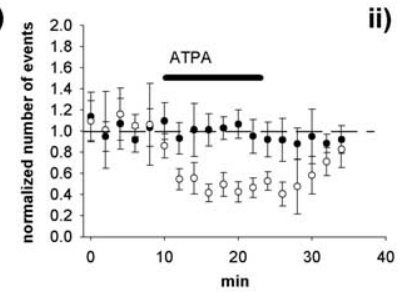

ii)
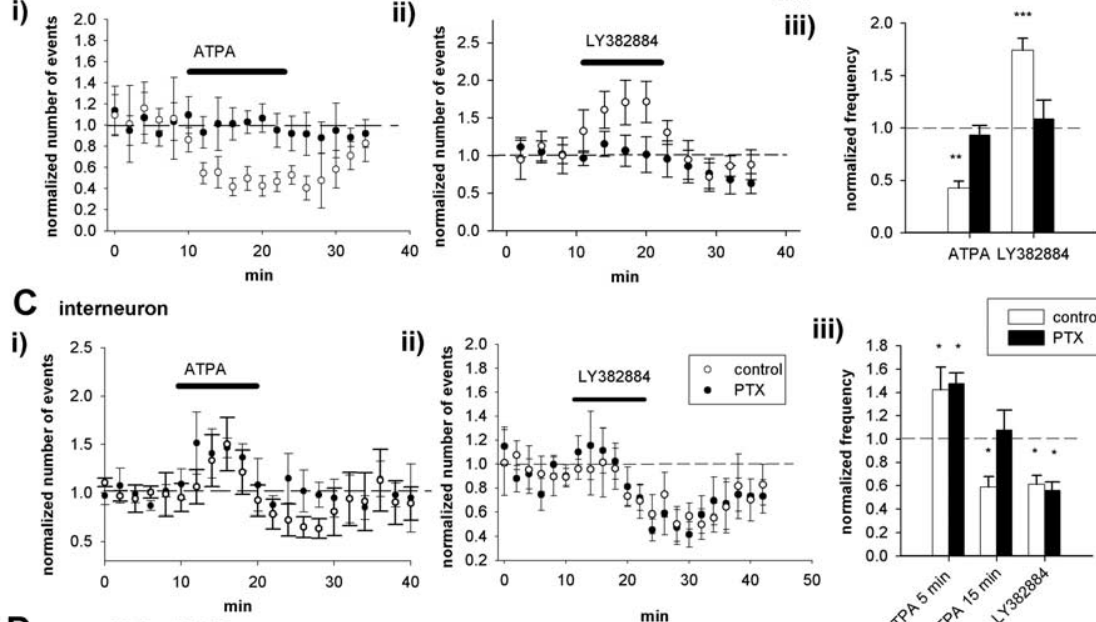

ii)
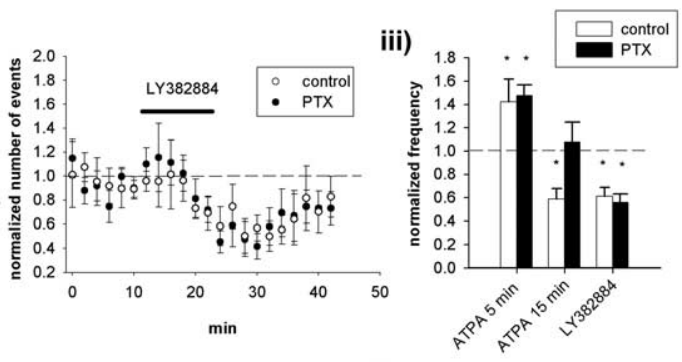

pyramidal cell, BIS
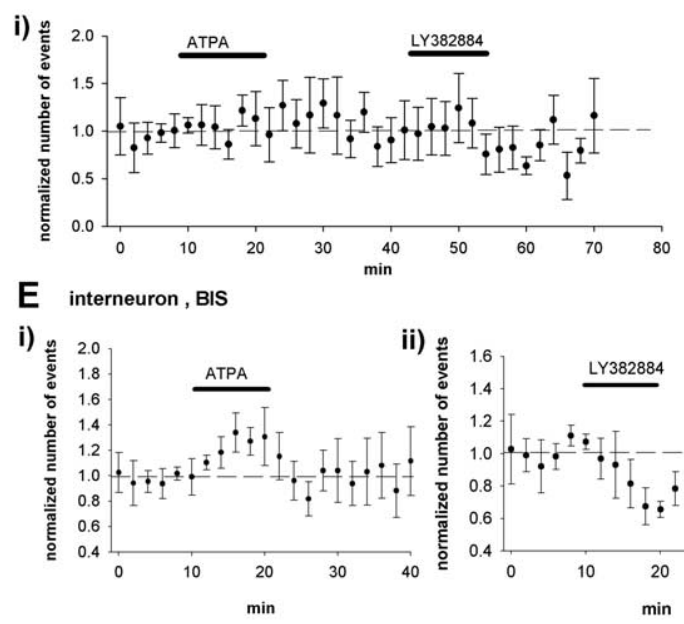

ii)
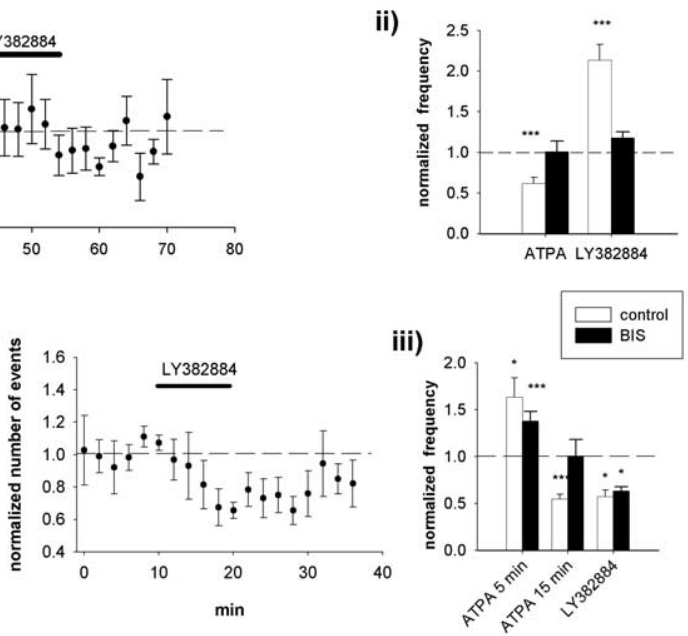

Figure 7. Inhibitory, but not facilitatory, actions of KARs on glutamate release are dependent on pertussis toxin-sensitive G-proteins and PKC. A, A control experiment demonstrating lack of effect of baclofen on IPSCs in PTX-preincubated slices. Monosynaptic IPSCS were evoked in CA1 neurons by afferent stimulation in the presence of CNQX or NBQX and D-AP-5. In control slices, $10 \mu \mathrm{m}$ baclofen caused a pronounced depression of IPSC amplitude, whereas in slices incubated in the presence of PTX, baclofen had no effect. Ai, Aii, Example traces ( $\mathbf{A i}$; average of 3 successive IPSCS, obtained at the time points indicated) and pooled data (Aii) from seven control and 10 PTX-pretreated slices. $\boldsymbol{B}$, Pooled data showing the effects of ATPA and LY382884 on the mEPSC frequency in CA3 pyramidal neurons in control-incubated slices (open circles; ATPA, $n=7 ; \mathrm{LY} 382884, n=7$ ) and the lack of effect in PTX-treated slices (filled circles; ATPA, $n=$ 8; LY382884, $n=11$ ). Bi, Bii, The number of events as a function of time, calculated in $180 \mathrm{~s}$ bins, and normalized to the baseline level before application of LY382884. Biii, The average change in mEPSC frequency in the presence of KAR-selective drugs, in control and PTX-pretreated slices. C, Equivalent data showing the effects of ATPA and LY382884 on the frequency of mEPSCs at CA3 interneurons in control incubated slices (ATPA, $n=8 ;$ LY382884, $n=7$ ) and in PTX-incubated slices (ATPA, $n=8 ;$ : LY382884, $n=9)$. D, Pooled data $(n=$ 7) showing the lack of effect of ATPA and LY382884 on mEPSC frequency in CA3 pyramidal cells in the presence of bisindolylmaleimide VIII (BIS) $(0.5 \mu \mathrm{m})$, a selective inhibitor for PKC. Di, The number of events as a function of time, calculated in 120 s bins. Dii, The average change in mEPSC frequency in the presence of ATPA $(n=8)$ and LY382884 $(n=7)$ in the presence ofBIS and under control conditions (ATPA, $n=$ 8; LY382884, $n=8)$. $E$, Equivalent data showing the effect of ATPA $(E i ; n=9)$ and LY382884 $(E i i ; n=6)$ on mEPSC frequency in CA3 interneurons in the presence of BIS and compared with control conditions(Eiii). ${ }^{* * *} p<0.005,{ }^{* *} p<0.01$, and ${ }^{*} p<0.05$ compared with baseline values.

frequency-dependent manner (Menendez de la Prida et al., 1999; Lamsa et al., 2000; Palva et al., 2000; Wells et al., 2000). Because the synaptic activation of KARs has been shown to bidirectionally regulate both L-glutamate and GABA release (Jiang et al., 2001;

Lauri et al., 2001a; Schmitz et al., 2001), we were interested in determining how KARs contribute to the modulation of the early network activity.

Activation of KARs by ATPA caused a substantial inhibition of synchronous network bursts in the neonatal hippocampus. This effect was associated with a large increase in the frequency of sIPSCs and depolarization of interneurons. ATPA had no effects on mIPSCs in either interneurons or pyramidal cells. Therefore, the regulation of sIPSCs in neonatal CA3 is presumably mediated via somatodendritic and/or axonal KARs on GABAergic interneurons, similar to what has been described in the adult hippocampus (Frerking et al., 1998; Cossart et al., 2001; Semyanov and Kullmann, 2001; Vignes, 2001; Khalilov et al., 2002). GABAergic transmission is critical in controlling the excitability and synchronization of the immature neuronal network (Lamsa et al., 2000; Wells et al., 2000). Thus, the increase in asynchronous GABAergic drive after KAR activation is likely to inhibit the network bursting. Furthermore, AMPA receptormediated transmission is necessary for driving the activity, because the AMPA receptorselective antagonist GYKI53655 completely blocks the network bursts (Bolea et al., 1999). Thus, activation of GluR5 subunitcontaining KARs suppresses the network activity by two parallel mechanisms: (1) increasing asynchronous GABAergic transmission and (2) attenuating AMPA receptor-mediated transmission in the pyramidal cells. Although ATPA transiently increased the occurrence of spontaneous EPSCs in interneurons, there was a parallel 10 -fold increase in the sIPSCs. This will impose a strong inhibitory action on the local network. Together, the shift in the balance between glutamatergic and GABAergic activity toward the latter one efficiently abolishes the synchronous activity of the network.

Interestingly, application of LY382884 resulted in a decrease in the frequency of network bursts, suggesting that endogenous activation of GluR5 subunitcontaining KARs is involved in the burst initiation. The mechanisms regulating the initiation of the network bursting are complicated and not fully understood. A key role is thought to be played by the excitatory synaptic input to interneurons, which is critical in controlling their excitability and synchronization (Khazipov et al., 1997; Lamsa et al., 2000). Apart from the synchronous network bursts, the endogenous activation of GluR5 subunit-containing KARs appeared to selectively regulate glutamatergic transmission, because LY382884 had no effect on sIPSCs, mIPSCs, or the 
holding current of interneurons. It is possible that local transient activation of KARs takes place in interneurons during intense activity such as the network bursts. Nevertheless, the regulation of action potential-independent glutamate release by KARs can fully explain the observed effects of LY382884 on spontaneous EPSCs in both pyramidal cells and interneurons. This mechanism might also underlie the observed effects on the network bursting, given their strong dependence on glutamatergic activity (Bolea et al., 1999; Lamsa et al., 2000). Thus, by inhibiting glutamatergic input to interneurons, LY382884 reduces the probability for synchronization of the interneuronal network, manifested as a decrease in the burst frequency. It is worth pointing out that, at P3-P6, the frequency of spontaneous glutamatergic events in interneurons was 20 -fold higher than in the pyramidal cells. Therefore, even if endogenously active KARs had the opposite effect on glutamate release to pyramidal neurons, their effect in the interneurons is dominating at the network level.

To summarize, our data show that the activation level of KARs is finely tuned to permit synchronized network activity in the neonatal hippocampus, because both increased activation and inhibition of GluR5 KARs disrupt the typical pattern on network activity. Apparently, these effects are dependent on distinct cellular mechanisms: activation of KARs vastly increases asynchronous inhibition and thus shunts the network, whereas inhibition of the KARs reduces interneuronal synchronization, therefore mitigating the build-up of network bursts.

Interestingly, KAR activation in the adult hippocampus causes oscillatory and epileptiform activity that was shown recently to be dependent on GluR5 and GluR6 KARs (Fisahn et al., 2004). Our data show that, in the neonate, endogenous activation of these receptors critically contributes to the synaptic drive, regulating the spontaneous network activity in a developmentally restricted period. In line with this, data from GluR5 ${ }^{-/-}$mice suggest altered network activity and a significant increase in the baseline frequency of sEPSCs in the CA3 pyramidal neurons compared with the wild types (M. Segerstråle, S. Lauri, C. Mulle, and T. Taira, unpublished results).

Developmental regulation of glutamate release by presynaptic KARs in the neonatal hippocampus

Although existence of presynaptic KARs in adult CA3 is well documented, there has hitherto been no evidence for presynaptic KAR regulating glutamate release at synaptic terminals to stra-

B i)
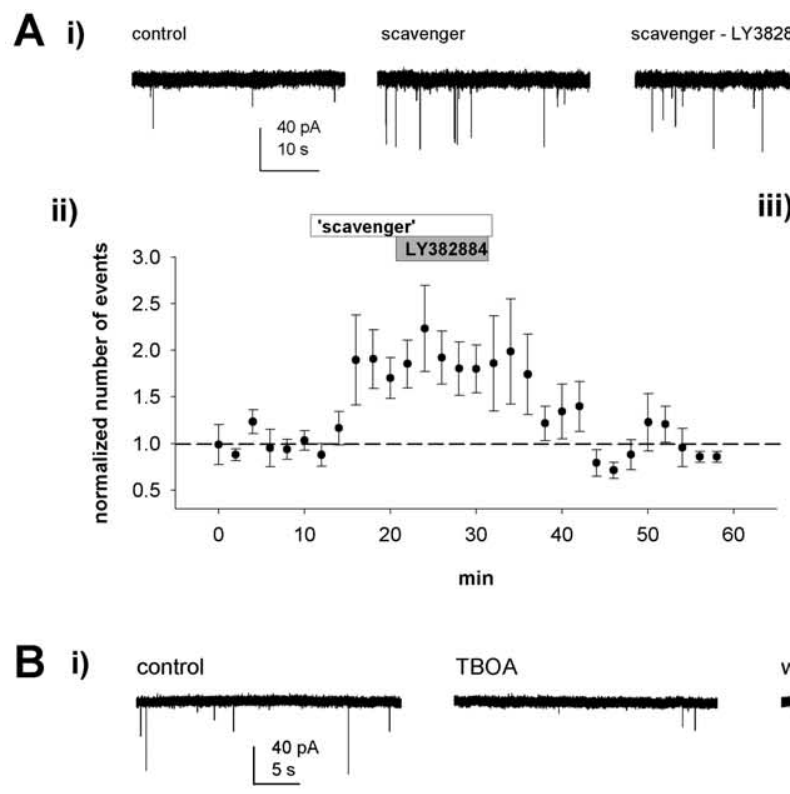

ii)

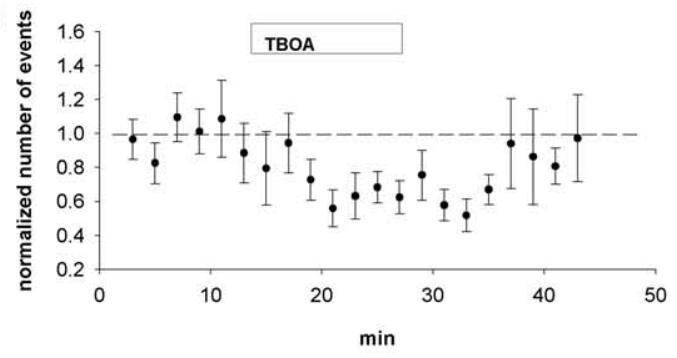

C

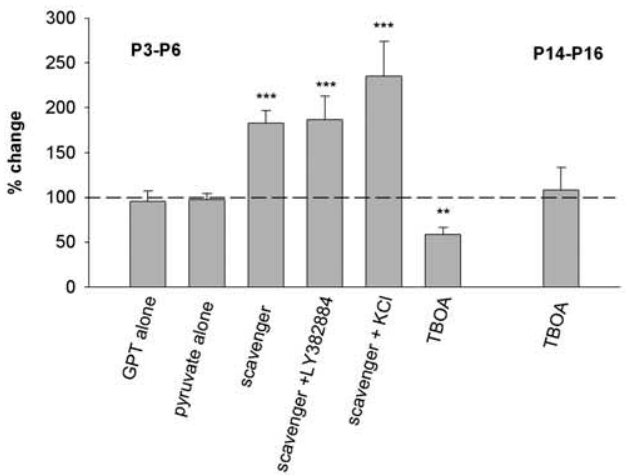

ii)
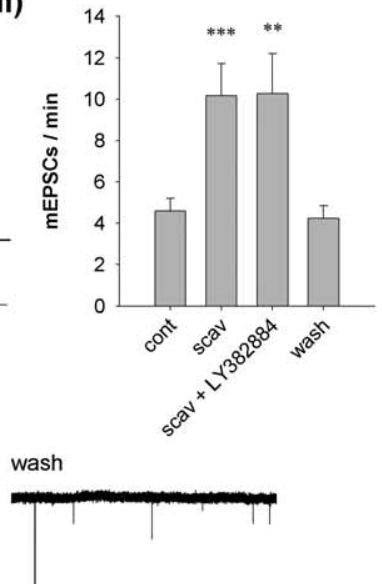

ii)

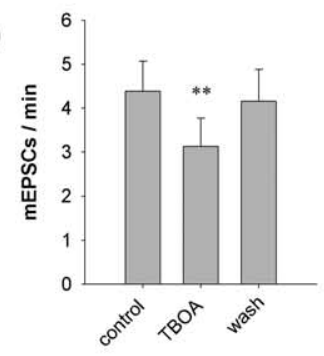

Figure 8. Ambient levels of L-glutamate activate presynaptic KARs in the neonatal hippocampus. $\boldsymbol{A}$, Single example (Ai) and pooled data $(n=12)$ show that a glutamate scavenger increases mEPSC frequency and fully occludes the effect of LY382884 at CA3 pyramidal neurons at P3-P6. Aii, The normalized number of events as a function of time, calculated in $120 \mathrm{~s}$ bins. Aiii, The average number of events per minute. scav, Scavenger. $\boldsymbol{B}$, Equivalent data for the effect of the glutamate uptake inhibitor DL-threo- $\beta$-benzyloxyaspartic acid (TBOA) (50 $\mu \mathrm{M})$ on mEPSC frequency at P3-P6 $(n=6)$. C, The graph shows pooled data of the mEPSC frequency in the presence of the two components of the glutamate scavenger GPT $(n=6)$ and pyruvate $(n=4)$ alone, as well as in the presence of the scavenger $(n=12), \mathrm{LY} 382884(n=12)$ and $4 \mathrm{mM} \mathrm{KCl}(n=7)$ together with the scavenger, and the glutamate uptake inhibitor TBOA $(50 \mu \mathrm{M})$ at P3-P6 $(n=6)$ and P14-P16 $(n=7)$. cont, Control; wash, Washout. Error bars represent SEM. ${ }^{* * *} p<0.005$ and ${ }^{* *} p<0.01$ compared with control. 
in the rat brain is lower at early stages of development. Second, differences in tissue morphology affect the diffusion of glutamate in the extracellular space (Sykova et al., 2000). These factors may contribute to the high levels of ambient glutamate providing the tonic activation of the presynaptic KARs, because the effects of LY382884 are fully occluded by the L-glutamate scavenger. The finding that these mechanisms are no longer active in 2-week-old animals is consistent with the developmental changes leading to more efficient clearance of L-glutamate from the vicinity of presynaptic KARs. Furthermore, maturation of the hippocampal circuitry might also involve changes in the presynaptic KARs per se. Alteration in the expression of GluR5 subunit-containing KARs during development has been reported previously (Bahn et al., 1994; Ritter et al., 2002). Developmental changes in the editing of GluR5 subunit-containing KARs (Bernard and Khrestchatisky, 1994; Lee et al., 2001) might also contribute to the alterations in the efficacy of the presynaptic KARs.

KARs have been shown to activate a metabotropic cascade to depress release at inhibitory synapses onto CA1 pyramidal neurons (Rodriguez-Moreno and Lerma, 1998). Furthermore, inhibition of glutamatergic transmission between CA3 and CA1 neurons by pharmacological activation of KARs involves a G-protein-dependent mechanism (Frerking et al., 2001). However, the physiological significance of the metabotropic action of KARs has been essentially unexplored. Here, we show that, in the neonate, their synaptic activation provides an inhibitory tone on L-glutamate release and that these receptors can be activated by ambient levels of L-glutamate present in the extracellular space. Furthermore, our data suggest that, at the same population of synapses, KARs can both facilitate and inhibit glutamate release via different signaling mechanisms. At glutamatergic synapses onto neonatal interneurons, activation of KARs transiently facilitated and then inhibited glutamate release via pertussis toxin and PKC-insensitive and -sensitive mechanisms, respectively.

The primary effect of presynaptic KARs is to inhibit glutamate release at synapses terminating at CA3 pyramidal cells and to facilitate release at synapses to interneurons. The most likely explanation resides in the diverse synaptic origin of the glutamatergic inputs into these cell types. In adults, CA3 interneurons receive 10 times more mossy fiber synapses than pyramidal cells (Acsady et al., 1998). However, the mossy fiber input to pyramidal cells develops predominantly during the second postnatal week in rats (Stirling and Bliss, 1978; Amaral and Dent, 1981; Marchal and Mulle, 2004); thus, at P3-P6, the recorded glutamatergic activity in the pyramidal neurons originates mainly from other sources, including the associational-commissural fibers and perforant pathway, both known to express presynaptic KARs (Chittajallu et al., 1996; Contractor et al., 2000). In area CA1, glutamatergic input onto interneurons develops earlier than onto pyramidal cells (Gozlan and Ben-Ari, 2003). The high frequency and the wide amplitude distribution of spontaneous EPSCs in interneurons suggests that similar sequential innervation of the cell types also takes place in the area CA3. Interestingly, in the interneurons, there was a trend suggesting a shift in the distribution toward higher-amplitude mEPSCs after kainate receptor activation and, correspondingly, toward smaller-amplitude mEPSCs in the presence of the KAR antagonist LY382884. In mature CA3, high-amplitude mEPSCs are thought to originate from the mossy fiber pathway, whereas low-amplitude events represent the input from the associate-commissural pathway and recurrent collaterals (Henze et al., 1997).

In summary, we show that neonatal KARs are critically involved in the regulation of emerging glutamatergic transmission and early network activity. They set a physiological tone on glutamatergic synapses that is only present during the first week of life, a time of intense activity-dependent plasticity and formation of synaptic contacts in the hippocampus.

\section{References}

Acsady L, Kamondi A, Sik A, Freund T, Buzsaki G (1998) GABAergic cells are the major postsynaptic targets of mossy fibers in the rat hippocampus. J Neurosci 18:3386-3403.

Amaral DG, Dent JA (1981) Development of the mossy fibers of the dentate gyrus. I. A light and electron microscopic study of the mossy fibres and their expansions. J Comp Neurol 95:51-86.

Anderson WW, Collingridge GL (2001) The LTP program: a data acquisition program for on-line analysis of long-term potentiation and other synaptic events. J Neurosci Methods 108:71-83.

Bahn S, Volk B, Wisden W (1994) Kainate receptor gene expression in the developing rat brain. J Neurosci 14:5525-5547.

Ben-Ari Y, Cherubini E, Corradetti R, Gaiarsa JL (1989) Giant synaptic potentials in immature rat CA3 hippocampal neurones. J Physiol (Lond) 416:303-325.

Bernard A, Khrestchatisky M (1994) Assessing the extent of RNA editing in the TMII regions of GluR5 and GluR6 kainate receptors during rat brain development. J Neurochem 62:2057-2060.

Bolea S, Avignone E, Berretta N, Sanchez-Andres JV, Cherubini E (1999) Glutamate controls the induction of GABA-mediated giant depolarizing potentials through AMPA receptors in neonatal rat hippocampal slices. J Neurophysiol 81:2095-2102.

Bortolotto ZA, Clarke VRJ, Delany CM, Parry MC, Smolders I, Vignes M, Ho KH, Miu P, Brinto BT, Fantaske R, Ogden A, Gates M, Ornstein PL, Lodge D, Bleakman D, Collingridge GL (1999) Kainate receptors are involved in synaptic plasticity. Nature 402:297-301.

Bortolotto ZA, Lauri SE, Isaac JTR, Collingridge GL (2003) Kainate receptors and the induction of mossy fibre long-term potentiation. Philos Trans R Soc Lond B Biol Sci 358:657-666.

Chittajallu R, Vignes M, Dev KK, Barnes JM, Collingridge GL, Henley JM (1996) Regulation of glutamate release by presynaptic kainate receptors in the hippocampus. Nature 379:78-81.

Clarke VRJ, Collingridge GL (2002) Characterisation of the effects of ATPA, $\mathrm{a} \mathrm{GLU}_{\mathrm{K5}}$ receptor selective agonist, on excitatory synaptic transmission in area CA1 of rat hippocampal slices. Neuropharmacology 42:889-902.

Clarke VRJ, Ballyk BA, Hoo KH, Mandelzys A, Pellizzari A, Bath CP, Thomas J, Sharpe EF, Davies CH, Ornstein PL, Schoepp DD, Kamboj RK, Collingridge GL, Lodge D, Bleakman D (1997) A hippocampal GluR5 kainate receptor regulating inhibitory synaptic transmission. Nature 389:599-603

Contractor A, Swanson GT, Sailer A, O'Gorman S, Heinemannm SF (2000) Identification of the kainate receptor subunits underlying modulation of excitatory synaptic transmission in the CA3 region of the hippocampus. J Neurosci 20:8269-8278.

Contractor A, Swanson G, Heinemann SF (2001) Kainate receptors are involved in short- and long-term plasticity at mossy fiber synapses in the hippocampus. Neuron 29:209-216.

Cossart R, Tyzio R, Dinocourt C, Esclapez M, Hirsch JC, Ben-Ari Y, Bernard C (2001) Presynaptic kainate receptors that enhance the release of GABA on CA1 hippocampal interneurons. Neuron 29:497-508.

Danbolt NC (2001) Glutamate uptake. Prog Neurobiol 65:1-105.

Delaney AJ, Jahr CE (2002) Kainate receptors differentially regulate release at two parallel fiber synapses. Neuron 36:475-482.

Fisahn A, Contractor A, Traub RD, Buhl EH, Heinemann SF, McBain CJ (2004) Distinct roles for the kainate receptor subunits GluR5 and GluR6 in kainate-induced hippocampal gamma oscillations. J Neurosci 24:9658-9668.

Fitzjohn SM, Bortolotto ZA, Palmer MJ, Doherty AJ, Ornstein PL, Schoepp DD, Kingston AE, Lodge D, Collingridge GL (1998) The potent mGlu receptor antagonist LY341495 identifies roles for both cloned and novel mGlu receptors in hippocampal synaptic plasticity. Neuropharmacology 37:1445-1458.

Frerking M, Malenka RC, Nicoll RA (1998) Synaptic activation of kainate receptors on hippocampal interneurons. Nat Neurosci 1:479-486.

Frerking M, Petersen CC, Nicoll RA (1999) Mechanisms underlying kainate receptor-mediated disinhibition in the hippocampus. Proc Natl Acad Sci USA 96:12917-12922. 
Frerking M, Schmitz D, Zhou Q, Johansen J, Nicoll RA (2001) Kainate receptors depress excitatory synaptic transmission at CA3 224 CA1 synapses in the hippocampus via a direct presynaptic action. J Neurosci 21:2958-2966.

Garaschuk O, Hanse E, Konnerth A (1998) Developmental profile and synaptic origin of early network oscillations in the CA1 region of rat neonatal hippocampus. J Physiol (Lond) 507:219-236.

Gozlan H, Ben-Ari Y (2003) Interneurons are the source and the targets of the first synapses formed in the rat developing hippocampal circuit. Cereb Cortex 13:684-692.

Groc L, Petanjek Z, Gustafsson B, Ben-Ari Y, Hanse E, Khazipov R (2002) In vivo blockade of neural activity alters dendritic development of neonatal CA1 pyramidal cells. Eur J Neurosci 16:1931-1938.

Henze DA, Card JP, Barrionuevo G, Ben-Ari Y (1997) Large amplitude miniature excitatory postsynaptic currents in hippocampal CA3 pyramidal neurons are of mossy fiber origin. J Neurophysiol 77:1075-1086.

Jiang L, Xu J, Nedergaard M, Kang J (2001) A kainate receptor increases the efficacy of GABAergic synapses. Neuron 30:503-513.

Kamiya H (2002) Kainate receptor-dependent presynaptic modulation and plasticity. Neurosci Res 42:1-6.

Kamiya H, Ozawa S (2000) Kainate receptor-mediated presynaptic inhibition at the mouse hippocampal mossy fibre synapse. J Physiol (Lond) 523:653-665.

Khalilov I, Hirsch J, Cossart R, Ben-Ari Y (2002) Paradoxical anti-epileptic effects of a GluR5 agonist of kainate receptors. J Neurophysiol $88: 523-527$

Khazipov R, Leinekugel X, Khalilov I, Gaiarsa JL, Ben-Ari Y (1997) Synchronization of GABAergic interneuronal network in CA3 subfield of neonatal rat hippocampal slices. J Physiol (Lond) 498:763-772.

Kidd FL, Coumis U, Collingridge GL, Crabtree JW, Isaac JTR (2002) A presynaptic kainate receptor is involved in regulating the dynamic properties of thalamocortical synapses during development. Neuron 34:635-646.

Kullmann DM (2001) Presynaptic kainate receptors in the hippocampus: slowly emerging from obscurity. Neuron 32:561-564.

Lahtinen H, Palva JM, Sumanen S, Voipio J, Kaila K, Taira T (2002) Postnatal development of rat hippocampal gamma rhythm in vivo. J Neurophysiol 88:1469-1474.

Lamsa K, Palva JM, Ruusuvuori E, Kaila K, Taira T (2000) Synaptic GABA(A) activation inhibits AMPA-kainate receptor-mediated bursting in the newborn (P0-P2) rat hippocampus. J Neurophysiol 83:359-366.

Lauri SE, Bortolotto ZA, Bleakman D, Ornstein PL, Lodge D, Isaac JTR, Collingridge GL (2001a) A critical role of a facilitatory presynaptic kainate receptor in mossy fiber LTP. Neuron 32:697-709.

Lauri SE, Delany C, Clarke VRJ, Bortolotto ZA, Ornstein PL, Isaac JTR, Collingridge GL (2001b) Synaptic activation of a presynaptic kainate receptor facilitates AMPA receptor-mediated synaptic transmission at hippocampal mossy fibre synapses. Neuropharmacology 41:907-915.

Lauri SE, Bortolotto ZA, Bleakman D, Ornstein PL, Lodge D, Isaac JTR, Collingridge GL (2003a) A role for $\mathrm{Ca}^{2+}$ stores in kainate-dependent synaptic facilitation and LTP at mossy fibre synapses in the hippocampus. Neuron 39:327-341.

Lauri SE, Lamsa K, Pavlov I, Riekki R, Johnston B, Molnar E, Rauvala H, Taira $\mathrm{T}$ (2003b) Activity blockade induces formation of functional synapses in the newborn rat hippocampus. Mol Cell Neurosci 22:107-117.

Lee CJ, Kong H, Manzini MC, Albuquerque C, Chao MV, MacDermott AB (2001) Kainate receptors expressed by a subpopulation of developing nociceptors rapidly switch from high to low $\mathrm{Ca}^{2+}$ permeability. J Neurosci 21:4572-4581.

Leinekugel X, Khazipov R, Cannon R, Hirase H, Ben-Ari Y, Buzsak G (2002) Correlated bursts of activity in the neonatal hippocampus in vivo. Science 296:2049-2052.

Lerma J (2003) Roles and rules of kainate receptors in synaptic transmission. Nat Rev Neurosci 4:481-495.
Luthi A, Schwyer L, Mateos JM, Gähwiler BH, McKinney RA (2001) NMDA receptor activation limits the number of synaptic connections during hippocampal development. Nat Neurosci 4:1102-1107.

Marchal C, Mulle C (2004) Postnatal maturation of mossy fibre excitatory transmission in mouse CA3 pyramidal cells: a potential role for kainate receptors. J Physiol 561:27-37.

Menendez de la Prida L, Sanchez-Andres JV (1999) Nonlinear frequencydependent synchronization in the developing hippocampus. J Neurophysiol 82:202-208.

Min M-Y, Rusakov DA, Kullmann DM (1998) Activation of AMPA, kainate and metabotropic receptors at hippocampal mossy fibre synapses: role of glutamate diffusion. Neuron 21:561-570.

Mulle C, Sailer A, Swanson GT, Brana C, O'Gorman S, Bettler B, Heinemann SF (2000) Subunit composition of kainate receptors in hippocampal interneurons. Neuron 28:475-484.

Overstreet LS, Pasternak JF, Colley A, Slater NT, Trommer BL (1997) Metabotropic glutamate receptor mediated long-term depression in developing hippocampus. Neuropharmacology 36:831-844.

Palva JM, Lamsa K, Lauri SE, Rauvala H, Kaila K, Taira T (2000) Fast network oscillations in the newborn rat hippocampus in vitro. J Neurosci 20:1170-1178.

Ritter LM, Vazquez DM, Meador-Woodruff JH (2002) Ontogeny of ionotropic glutamate receptor subunit expression in the rat hippocampus. Brain Res Dev Brain Res 139:227-236.

Rodriguez-Moreno A, Lerma J (1998) Kainate receptor modulation of GABA release involves a metabotropic function. Neuron 20:1211-1218.

Rusakov DA, Kullmann DM (1998) Extrasynaptic glutamate diffusion in the hippocampus: ultrastructural constraints, uptake and receptor activation. J Neurosci 18:3158-3170.

Scanziani M, Salin PA, Vogt KE, Malenka RC, Nicoll RA (1997) Usedependent increases in glutamate concentration activate presynaptic metabotropic glutamate receptors. Nature 385:630-634.

Schmitz D, Frerking M, Nicol RA (2000) Synaptic activation of presynaptic kainate receptors on hippocampal mossy fiber synapses. Neuron 27:327-338.

Schmitz D, Mellor J, Nicoll RA (2001) Presynaptic kainate receptor mediation of frequency facilitation at hippocampal mossy fiber synapses. Science 291:1972-1976.

Schmitz D, Mellor J, Breustedt J, Nicoll RA (2003) Presynaptic kainate receptors impart an associative property to hippocampal mossy fiber longterm potentiation. Nat Neurosci 6:1058-1063.

Semyanov A, Kullmann DM (2001) Kainate receptor-dependent axonal depolarization and action potential initiation in interneurons. Nat Neurosci 4:718-723.

Stirling RV, Bliss TV (1978) Hippocampal mossy fiber development at the ultrastructural level. Prog Brain Res 48:191-198.

Sykova E, Mazel T, Vargova L, Vorisek I, Prokopova, Kubinova S (2000) Extracellular space diffusion and pathological states. Prog Brain Res 125:155-178.

Toullec D, Pianetti P, Coste H, Bellevergue P, Grand-Perret T, Ajakane M, Baudet V, Boissin P, Boursier E, Loriolle F, Duhamel L, Charon D, Kirilovsky J (1991) The bisindolylmaleimide GF 109203X is a potent and selective inhibitor of protein kinase C. J Biol Chem 266:15771-15781.

Vignes M (2001) Regulation of spontaneous inhibitory synaptic transmission by endogenous glutamate via non-NMDA receptors in cultured rat hippocampal neurons. Neuropharmacology 40:737-748.

Wells JE, Porter JT, Agmon A (2000) GABAergic inhibition suppresses paroxysomal network activity in the neonatal rodent hippocampus and neocortex. J Neurosci 20:8822-8830.

Zhang LI, Poo MM (2001) Electrical activity and development of neural circuits. Nat Neurosci 4:1207-1214. 